H. Naitoh

Nagoya Math. J.

Vol. 90 (1983), 85-117

\title{
PARALLEL SUBMANIFOLDS OF COMPLEX SPACE FORMS I
}

\author{
HIROO NAITOH*) \\ Dedicated to Professor Kentaro Murata on his sixtieth birthday
}

\section{Introduction}

Complete parallel submanifolds of a real space form of constant sectional curvature $k$ have been completely classified by Ferus [3] when $k \geqq$ 0 , and by Takeuchi [19] when $k<0$. A complex space form is by definition a $2 n$-dimensional simply connected Hermitian symmetric space of constant holomorphic sectional curvature $c$ and will be denoted by $\bar{M}^{n}(c)$. The complex space form $\bar{M}^{n}(0)$ is isometric to the Euclidean space $R^{2 n}$ and so complete parallel submanifolds of $\bar{M}^{n}(0)$ are known by Ferus [3], $k=0$. Assume that $c \neq 0$. Then we know that a parallel submanifold of $\bar{M}^{n}(c)$ is Kählerian or totally real. Complete Kählerian parallel submanifolds of $\bar{M}^{n}(c)$ have been completely classified by Nakagawa-Takagi [13] when $c>0$, and by Kon [9] when $c<0$. Recently, Naitoh [11] has shown that the classification of $n$-dimensional complete totally real parallel submanifolds of $\bar{M}^{n}(c), c>0$, is reduced to that of certain cubic forms of $n$-variables and Naitoh-Takeuchi [12] has classified these submanifolds by the theory of symmetric bounded domains of tube type.

In the present article we will study the classification of complete parallel submanifolds of $\bar{M}^{n}(c), \quad c \neq 0$.

In the present paper $\mathrm{I}$, we will show that a parallel submanifold of $\bar{M}^{n}(c), \quad c \neq 0$, is one of the following three types:

(a) Kählerian submanifold,

(b) totally real submanifold which is contained in a totally real totally geodesic submanifold,

(c) totally real submanifold which is contained in a totally geodesic Kählerian submanifold whose dimension is twice of the dimension of the submanifold (Theorem 2.4).

Received December 14, 1981.

*) Partially supported by the Yukawa fellowship. 
This implies that the classification of complete parallel submanifolds of $\bar{M}^{n}(c), c>0$, is reduced to those of Nakagawa-Takagi [13], Ferus [3], $k>0$, and Naitoh-Takeuchi [12]. As application of our classification we will completely classify complete $\lambda$-isotropic parallel submanifolds of $\bar{M}^{n}(c)$, $c>0$, which the author has studied ealier in [10], [11] (Corollary 3.4). Now, if $c<0$, the classifications of complete parallel submanifolds of types (a), (b) are reduced to those of Kon [9], Takeuchi [19] respectively.

In the section 4 we will study the "complete inverse" of an $r$-dimensional complete totally real parallel submanifold of $\bar{M}^{r}(c), c \neq 0$. The complete inverse is an $(r+1)$-dimensional complete totally real parallel submanifold of a pseudo-Hermitian space $\boldsymbol{E}^{r+1}$ (Proposition 4.1).

In the section 5 we will define "orthogonal Jordan triple system" and "orthogonal symmetric graded Lie algebra". These notions may be regarded as extensions of non-degenerate Jordan triple system and semisimple symmetric graded Lie algebra respectively. And two notions have a natural one-to-one correspondence (Theorem 5.4). Moreover we will construct a parallel submanifold of a pseudo-Euclidean space from an orthogonal symmetric graded Lie algebra satisfying a certain condition (Theorem 5.7).

In the forthcoming paper II we will construct an orthogonal Jordan triple system and an orthogonal symmetric graded Lie algebra associated with a complete inverse submanifold and show that the complete inverse submanifold is equivalent to the parallel submanifold constructed from the orthogonal symmetric graded Lie algebra. And we will classify $r$ dimensional complete totally real parallel submanifolds of $\bar{M}^{r}(c), c<0$, by determining orthogonal symmetric graded Lie algebras associated with complete inverses.

The author wishes to express his hearty thanks to Professor S. Murakami for useful comments and to Professor M. Takeuchi for much information.

\section{$\S 1$. Preliminaries}

Let $M, \bar{M}$ be connected pseudo-riemannian manifolds and $f$ an isometric immersion of $M$ into $\bar{M}$. Throughout this paper we will identify a vector $X$ of $M$ with a vector $f_{*}(X)$ of $\bar{M}$. The pseudo-riemannian metrics on $M, \bar{M}$ are denoted by the same notation $<,>$. Let $\nabla, \bar{\nabla}$ be the LeviCivita connections on $M, \bar{M}$ respectively. The metric and the connection 
on the pull back $f^{-1} T(\bar{M})$ of the tangent bundle $T(\bar{M})$ of $\bar{M}$, induced from $\langle$, $\rangle$ and $\bar{\nabla}$, are also denoted by $\langle$, $\rangle$ and $\bar{\nabla}$. The pull back $f^{-1} T(\bar{M})$ is orthogonally decomposed into the sum of tangent bundle $T(M)$ of $M$ and normal bundle $N(M)$ for $f$. Let $D$ be the normal connection on $N(M), \sigma_{f}$ the second fundamental form of $f$, and $A$ the shape operator of $f$. Then we have the formulas:

$$
\begin{gathered}
\bar{\nabla}_{X} Y=\nabla_{X} Y+\sigma_{f}(X, Y), \\
\bar{\nabla}_{X} \zeta=-A_{\zeta} X+D_{X} \zeta \\
\left\langle A_{\zeta} X, Y\right\rangle=\left\langle\sigma_{f}(X, Y), \zeta\right\rangle, \sigma_{f}(X, Y)=\sigma_{f}(Y, X)
\end{gathered}
$$

for vector fields $X, Y$ of $M$ and a normal vector field $\zeta$. We define

$$
\left(\nabla^{*} \sigma_{f}\right)(X, Y, Z)=\left(\nabla_{X}^{*} \sigma_{f}\right)(Y, Z)=D_{X} \sigma_{f}(Y, Z)-\sigma_{f}\left(\nabla_{X} Y, Z\right)-\sigma_{f}\left(Y, \nabla_{X} Z\right)
$$

for vector fields $X, Y, Z$ of $M$. The isometric immersion $f$ is called parallel if $\nabla^{*} \sigma_{f}=0$, and the image $f(M)$ of a parallel imbedding $f$ is called a parallel submanifold of $\bar{M}$. Denote by $R, \bar{R}, R^{\perp}$ the curvature tensors for $\nabla, \bar{\nabla}$, $D$ respectively and by $\{*\}^{\perp}$ the normal component of $*$. Then we have the Gauss-Codazzi-Ricci equations for an isometric immersion $f$ :

$$
\begin{aligned}
\langle\bar{R}(X, Y) Z, W\rangle= & \langle R(X, Y) Z, W\rangle+\left\langle\sigma_{f}(X, Z), \sigma_{f}(Y, W)\right\rangle \\
& -\left\langle\sigma_{f}(X, W), \sigma_{f}(Y, Z)\right\rangle, \\
\{\bar{R}(X, Y) Z\}^{\perp}=\left(\nabla_{X}^{*} \sigma_{f}\right)(Y, Z)-\left(\nabla_{Y}^{*} \sigma_{f}\right)(X, Z), & \left\langle R^{\perp}(X, Y) \zeta, \xi\right\rangle-\left\langle\left[A_{\zeta}, A_{\xi}\right] X, Y\right\rangle
\end{aligned}
$$

for vector fields $X, Y, Z, W$ of $M$ and normal vector fields $\zeta, \xi$.

Now, for a point $p$ of an $n$-dimensional pseudo-riemannian manifold $M$, there exists a basis $\left\{e_{1}, \cdots, e_{k}, e_{k+1}, \cdots, e_{n}\right\}$ of the tangent space $T_{p}(M)$ such that

$$
\left\langle e_{i}, e_{j}\right\rangle=\left\{\begin{aligned}
-1, & 1 \leqq i=j \leqq k \\
1, & k+1 \leqq i=j \leqq n \\
0, & i \neq j
\end{aligned}\right.
$$

Here note that the non-negative integer $k$ is independent of a point $p$. The pair $(k, n-k)$ is called the signature of the pseudo-riemannian manifold $M$, and $\left\{e_{1}, \cdots, e_{n}\right\}$ an orthonormal basis of $T_{p}(M)$.

Let $f$ be an isometric immersion of an $n$-dimensional pseudo-rieman- 
nian manifold $M$ of signature $(k, n-k)$ into a pseudo-riemannian manifold $\bar{M}$. We define the mean curvature vector field $\eta_{f}$ for $f$ as follows:

$$
n \cdot \eta_{f}(p)=-\sum_{i=1}^{k} \sigma_{f}\left(e_{i}, e_{i}\right)+\sum_{i=k+1}^{n}\left(e_{i}, e_{i}\right)
$$

for an orthonormal basis $\left\{e_{1}, \cdots, e_{n}\right\}$ of $T_{p}(M)$. The isometric immersion $f$ is called minimal if $\eta_{f}=0$. It is called totally umbilical if

(1) the mean curvature vector field $\eta_{f}$ is nowhere zero and $\sigma_{f}(X, Y)=$ $\langle X, Y\rangle \eta_{f}$ for vector fields $X, Y$ of $M$, and

(2) $\eta_{f}$ is parallel; $D \eta_{f}=0$.

The image $f(M)$ of a totally umbilical isometric imbedding $f$ is called a totally umbilical submanifold of $\bar{M}$. Here note that a totally umbilical isometric immersion is always parallel.

Let $f_{i}: M_{i} \rightarrow \bar{M}_{i}, 1 \leqq i \leqq s$, be isometric immersions of $n_{i}$-dimensional pseudo-riemannian manifolds $M_{i}$ into pseudo-riemannian manifolds $\bar{M}_{i}$. Denote by $\sigma_{f i}, \eta_{f_{i}}$ the second fundamental form and the mean curvature vector field of $f_{i}$ respectively. The product isometric immersion $f=f_{1} \times$ $\cdots \times f_{s}$ of the product pseudo-riemannian manifold $M=M_{1} \times \cdots \times M_{s}$ into the product pseudo-riemannian manifold $\bar{M}=\bar{M}_{1} \times \cdots \times \bar{M}_{s}$ is defined by

$$
f\left(p_{1}, \cdots, p_{s}\right)=\left(f_{1}\left(p_{1}\right), \cdots, f_{s}\left(p_{s}\right)\right)
$$

for $p_{i} \in M_{i}, 1 \leqq i \leqq s$. Then we have

$$
\begin{gathered}
\sigma_{f}\left(X_{1}+\cdots+X_{s}, Y_{1}+\cdots+Y_{s}\right)=\sigma_{f_{1}}\left(X_{1}, Y_{1}\right)+\cdots+\sigma_{f_{s}}\left(X_{s}, Y_{s}\right), \\
n \cdot \eta_{f}=n_{1} \cdot \eta_{f_{1}}+\cdots+n_{s} \cdot \eta_{f_{s}}
\end{gathered}
$$

for vector fields $X_{i}, Y_{i}$ of $M_{i}, 1 \leqq i \leqq s$. Therefore, $f$ is parallel (resp. minimal) if and only if each $f_{i}$ is parallel (resp. minimal). Moreover, $\eta_{f}$ is parallel if and only if each $\eta_{f_{i}}$ is parallel.

Lemma 1.1 (cf. Takeuchi [19]). Let $M, M^{\prime}, \bar{M}$, be pseudo-riemannian manifolds. Let $f^{\prime}: M \rightarrow M^{\prime}$ be an isometric immersion, $f^{\prime \prime}: M^{\prime} \rightarrow \bar{M}$ a totally geodesic or totally umbilical isometric immersion and let $f=f^{\prime \prime} \circ f^{\prime}$ be the composition of $f^{\prime}$ and $f^{\prime \prime}$. Then

(1) $\sigma_{f}(X, Y)=\sigma_{f^{\prime}}(X, Y)+\langle X, Y\rangle \eta_{f^{\prime \prime}}, \quad \eta_{f}=\eta_{f^{\prime}}+\eta_{f^{\prime \prime}} \circ f^{\prime}$, $D_{X} \eta_{f}=D_{X}^{\prime} \eta_{f^{\prime}}, \quad A_{\eta_{f}}=A_{\eta_{f}}^{\prime}+\left\langle\eta_{f^{\prime \prime}}, \eta_{f^{\prime \prime}}\right\rangle \mathrm{id}$

for vector fields $X, Y$ of $M$ (Here $D^{\prime}, A^{\prime}$ denote the normal connection and the shape operator for $f^{\prime}$ respectively), 
(2) $\eta_{f}$ is parallel if and only if $\eta_{f^{\prime}}$ is parallel,

(3) $f$ is parallel if and only if $f^{\prime}$ is parallel.

Proof. See [19, Lemma 1.1].

q.e.d.

\section{§2. The reduction theorem}

In this section we study parallel isometric immersions of riemannian manifolds $M$ into a complex space form $\bar{M}^{n}(c), c \neq 0$. (dim $\left.M \geqq 2\right)$

Let $\bar{M}$ be a Kählerian manifold and denote by $J$ the complex structure. An isometric immersion $f$ of a riemannian manifold $M$ into $\bar{M}$ is called Kählerian (resp. totally real) if $J T_{p}(M)=T_{p}(M)$ (resp. $J T_{p}(M) \subset$ $\left.N_{p}(M)\right)$ for $p \in M$. If $f$ is Kählerian, $M$ is a Kählerian manifold such that $f: M \rightarrow \bar{M}$ is a holomorphic isometric immersion.

Let $f: M \rightarrow \bar{M}$ be an isometric immersion of a riemannian manifold $M$ into a riemannian manifold $\bar{M}$. For a point $p \in M$, the first normal space $N_{p}^{1}(M)$ and the first osculating space $O_{p}^{1}(M)$ at $p$ are defined by

$$
N_{p}^{1}(M)=\left\{\sigma_{f}(X, Y): X, Y \in T_{p}(M)\right\}_{R}, \quad O_{p}^{1}(M)=T_{p}(M) \oplus N_{p}^{1}(M)
$$

where \{\}$_{\boldsymbol{R}}$ means the $R$-span of \{\} . Let $\bar{M}$ be a riemannian symmetric space. A linear subspace $V \subset T_{p}(M)$ is called a Lie triple system if $\bar{R}_{p}(X, Y) Z$ $\in V$ for $X, Y, Z \in V$. For a lie triple system $V$ there is a unique complete totally geodesic submanifold $N$ of $\bar{M}$ such that $p \in N, T_{p}(N)=V$ (cf. Helgason [6]).

Lemma 2.1. Let $f$ be a parallel isometric immersion of a riemannian manifold $M$ into a complex space form $\bar{M}^{n}(c), c \neq 0$. Then the first osculating space $O_{p}^{1}(M)$ is a Lie triple system in $T_{p}\left(\bar{M}^{n}(c)\right)$ for a point $p \in M$. Moreover, the following cases occur:

(a) The immersion $f$ is Kählerian and the subspace $O_{p}^{1}(M)$ is J-invariant, i.e., $J O_{p}^{1}(M)=O_{p}^{1}(M)$,

(b) The immersion $f$ is totally real and the subspace $O_{p}^{1}(M)$ is totally real, i.e., $J O_{p}^{1}(M)$ and $O_{p}^{1}(M)$ are orthogonal,

(c) The immersion $f$ is totally real and the subspace $O_{p}^{1}(M)$ is J-invariant.

Here if the subspace $O_{p}^{1}(M)$ is $J$-invariant (resp, totally real), the complete totally geodesic submanifold $N$ defined by $O_{p}^{1}(M)$ is a complex space form $\bar{M}^{r}(c)$ (resp. a real projective space $R^{s}(c / 4)$ or a real hyperbolic space $R H^{s}(c / 4)$ of constant sectional curvature c/4 according as $c>0, c<0$ respectively). 
Proof. A parallel isometric immersion of a riemannian manifold (dim $\geqq 2$ ) into $\bar{M}^{n}(c), c \neq 0$, is Kählerian or totally real (cf. Chen-Ogiue [1]) and the first osculating spaces are Lie triple systems (Naitoh [10]). Since a totally geodesic submanifold is parallel, the submanifold $N \subset \bar{M}^{n}(c)$ defined by $O_{p}^{1}(M)$ is Kählerian or totally real. This implies that $O_{p}^{1}(M)$ is $J$-invariant or totally real. If $f$ is Kählerian, we have $J T_{p}(M)=T_{p}(M)$ and $\sigma_{f}(J X, Y)=J \sigma_{f}(X, Y)$ for $X, Y \in T_{p}(M)$, and thus $O_{p}^{1}(M)$ is $J$-invariant.

Now it is known that a complete Kählerian (resp. totally real) totally geodesic submanifold of $\bar{M}^{n}(c)$ is $\bar{M}^{r}(c)$ (resp. $\boldsymbol{R} \boldsymbol{P}^{s}(c / 4)$ or $\boldsymbol{R} H^{s}(c / 4)$ according as $c>0, c<0$ respectively). This completes our proof. q.e.d.

Let $\bar{M}$ be a Kählerian manifold and denote by $J$ the complex structure. Let $f: M \rightarrow \bar{M}$ be a totally real isometric immersion of a riemannian manifold $M$ into $\bar{M}$. The normal space $N_{p}(M), p \in M$, is decomposed into the sum of subspace $J T_{p}(M)$ and its orthogonal complement $\left\{J T_{p}(M)\right\}^{\perp}$. Denote by $\sigma_{f}^{T}(X, Y)\left(\operatorname{resp} . \sigma_{f}^{\perp}(X, Y)\right)$ the $J T_{p}(M)$-component $\left(\operatorname{resp} .\left\{J T_{p}(M)\right\}^{\perp}\right.$ component) of $\sigma_{f}(X, Y)$ for $X, Y \in T_{p}(M)$. The $T(M)$-valued symmetric tensor $J \sigma_{f}^{T}$ is defined by

$$
\left(J \sigma_{f}^{T}\right)(X, Y)=J\left(\sigma_{f}^{T}(X, Y)\right)
$$

for vector fields $X, Y$ of $M$. If we identify tangent spaces $T_{p}(M)$ with cotangent spaces $T_{p}^{*}(M)$ through the riemannian metric on $M$, the tensor $J \sigma_{f}^{T}$ is a covariant tensor of degree 3 .

Lemma 2.2. Let $\bar{M}$ and $f$ be as above. Then,

(1) $J \sigma_{f}^{T}$ is a symmetric tensor of degree 3.

Moreover, if the totally real isometric immersion $f$ is parallel,

(2) the tensor $J \sigma_{f}^{T}$ is parallel, i.e., $\nabla\left(J \sigma_{f}^{T}\right)=0$,

(3) $\left\langle\sigma_{f}^{\perp}(X, W), J \sigma_{f}^{\perp}(Y, Z)\right\rangle=0$

for vector fields $X, Y, Z, W$ of $M$.

Proof. The claim (1) is the result of Lemma 2.4 in [10]. We show the claims (2), (3). For vector fields $X, Y, Z, W$ of $M$ we have

$$
\begin{aligned}
\left\langle\nabla_{X}\left(J \sigma_{f}^{T}(Y, Z)\right), W\right\rangle \\
\quad=\left\langle\bar{\nabla}_{X}\left(J \sigma_{f}^{T}(Y, Z)\right), W\right\rangle \\
\quad=-\left\langle\bar{\nabla}_{X}\left(\sigma_{f}^{T}(Y, Z)\right), J W\right\rangle \\
\quad=-\left\langle\bar{\nabla}_{X}\left(\sigma_{f}(Y, Z)\right), J W\right\rangle+\left\langle\bar{\nabla}_{X}\left(\sigma_{f}^{\perp}(Y, Z)\right), J W\right\rangle \\
\quad=-\left\langle D_{X}\left(\sigma_{f}(Y, Z)\right), J W\right\rangle-\left\langle\sigma_{f}^{\perp}(Y, Z), \bar{\nabla}_{X} J W\right\rangle
\end{aligned}
$$




$$
\begin{aligned}
&=-\left\langle\sigma_{f}\left(\nabla_{X} Y, Z\right)+\sigma_{f}\left(Y, \nabla_{X} Z\right), J W\right\rangle+\left\langle J \sigma_{f}^{\perp}(Y, Z), \bar{\nabla}_{X} W\right\rangle \quad \text { by the parallelity of } f, \\
&=-\left\langle\sigma_{f}^{T}\left(\nabla_{X} Y, Z\right)+\sigma_{f}^{T}\left(Y, \nabla_{X} Z\right), J W\right\rangle+\left\langle J \sigma_{f}^{\perp}(Y, Z), \sigma_{f}(X, W)\right\rangle \text { by }(1.1), \\
&=\left\langle J \sigma_{f}^{T}\left(\nabla_{X} Y, Z\right)+J \sigma_{f}^{T}\left(Y, \nabla_{X} Z\right), W\right\rangle+\left\langle J \sigma_{f}^{\perp}(Y, Z), \sigma_{f}^{\perp}(X, W)\right\rangle
\end{aligned}
$$

since the subspace $\left\{J T_{p}(M)\right\}^{\perp}$ is $J$-invariant. Hence we have

$$
\left\langle\left\{\nabla_{X}\left(J \sigma_{f}^{T}\right)\right\}(Y, Z), W\right\rangle=\left\langle\sigma_{f}^{\perp}(X, W), J \sigma_{f}^{\frac{1}{f}}(Y, Z)\right\rangle .
$$

Since $J \sigma_{f}^{T}$ is a symmetric tensor of degree 3, the equation (2.1) means that $\nabla\left(J \sigma_{f}^{T}\right)$ is a symmetric tensor of degree 4 . Moreover we have

$$
\left\langle\left\{\nabla_{X}\left(J \sigma_{f}^{T}\right)\right\}(X, X), X\right\rangle=\left\langle\sigma_{f}^{\perp}(X, X), J \sigma_{f}^{\perp}(X, X)\right\rangle=0
$$

for a vector field $X$ of $M$, and thus $\nabla\left(J \sigma_{f}^{T}\right)=0$. Together with (2.1) our claim (3) is proved.

q.e.d.

Let $\bar{M}$ be a riemannian manifold and $c(t)$ a curve in $\bar{M}$ defined on an open interval $I \ni 0$ and parametrized by arc-length. The curve $c(t)$ is called a Frenet curve in $\bar{M}$ of osculating rank $r(\geqq 1)$ if for all $t \in I$ its higher order derivatives

$$
\dot{c}(t)=\left(\bar{\nabla}_{\partial / \partial t}^{0} \dot{c}\right)(t),\left(\bar{\nabla}_{\partial / \partial t} \dot{c}\right)(t), \cdots,\left(\bar{\nabla}_{\partial / \partial t}^{r-1} \dot{c}\right)(t)
$$

are linearly independent but

$$
\dot{c}(t)=\left(\bar{\nabla}_{\partial / \partial t}^{0} \dot{c}\right)(t),\left(\bar{\nabla}_{\partial / \partial t} \dot{c}\right)(t), \cdots,\left(\bar{\nabla}_{\partial / \partial t}^{r} \dot{c}\right)(t)
$$

are linearly dependent in $T_{c(t)}(\bar{M})$. Then there exist unique positive $C^{\infty}$ functions $\kappa_{1}(t), \cdots, \kappa_{r-1}(t)$ on $I$ and unique orthonormal $C^{\infty}$-vector fields $V_{1}(t), \cdots, V_{r}(t)$ along the curve $c(t)$ such that

$$
\left\{\begin{array}{l}
\dot{c}(t)=V_{1}(t),\left(\bar{\nabla}_{\partial / \partial t} V_{1}\right)(t)=\kappa_{1}(t) V_{2}(t), \\
\left(\bar{\nabla}_{\partial / \partial t} V_{2}\right)(t)=-\kappa_{1}(t) V_{1}(t)+\kappa_{2}(t) V_{3}(t), \\
\quad \vdots \\
\left(\bar{\nabla}_{\partial / \partial t} V_{r-1}\right)(t)=-\kappa_{r-2}(t) V_{r-2}(t)+\kappa_{r-1}(t) V_{r}(t), \\
\left(\bar{\nabla}_{\partial / \partial t} V_{r}\right)(t)=-\kappa_{r-1}(t) V_{r-1}(t) .
\end{array}\right.
$$

Here we call $\kappa_{j}(t), 1 \leqq j \leqq r-1$, the Frenet curvature functions of $c(t)$, $\left\{V_{j}(t) ; 1 \leqq j \leqq r\right\}$ the Frenet $r$-frame along $c(t)$, and the equations (2.2) the Frenet formulas. For a given integer $r(\geqq 1)$ and given positive $C^{\infty}$-functions $\kappa_{1}(t), \cdots, \kappa_{r-1}(t)$ on $I$, the Frenet formulas may be regarded as a system of differential equations with variables $c, V_{1}, \cdots, V_{r}$. It is known 
that this system has a unique local solution for given initial conditions; a point $c(0)=p \in \bar{M}$ and an orthonormal $r$-frame $\left\{V_{j}(0)=V_{j} ; 1 \leqq j \leqq r\right\}$ of $T_{p}(\bar{M})$. If the riemannian manifold $\bar{M}$ is complete, the Frenet curve $c(t)$ is defined for $-\infty<t<+\infty$ (cf. [2], [17]).

Lemma 2.3 (Strübing [17]). Let $f: M \rightarrow \bar{M}$ be a parallel isometric immersion of a riemannian manifold $M$ into a riemannian manifold $\bar{M}$. If $\gamma(t)$ is a geodesic in $M$ parametrized by arc-length, the composition curve $(f \circ \gamma)(t)$ is a Frenet curve in $\bar{M}$.

TheOREM 2.4. Let $M$ be a complete riemannian manifold and $f$ a parallel isometric immersion of $M$ into a complex space form $\bar{M}^{n}(c), c \neq 0$. Then there exists a unique complete totally geodesic submanifold $N$ of $\bar{M}^{n}(c)$ such that $f(M) \subset N, T_{q}(N)=O_{q}^{1}(M), q \in M$.

Moreover, the following cases occur:

(a) The manifold $M$ and the immersion $f$ are Kählerian, and the submanifold $N$ is $\bar{M}^{r}(c)$,

(b) The immersion $f$ is totally real and the submanifold $N$ is a real projective space $R P^{s}(c / 4)$ or a real hyperbolic space $R H^{s}(c / 4)$ according as $c>0, c<0$ respectively,

(c) The immersion $f$ is totally real and the submanifold $N$ is $\bar{M}^{r}(c)$ with $r=\operatorname{dim} M$.

Proof. Fix a point $p \in M$. By Lemma 2.1 the first osculating space $O_{p}^{1}(M)$ is a Lie triple system, and thus defines a unique complete totally geodesic submanifold $N$ such that $p \in N, T_{p}(N)=O_{p}^{1}(M)$. Let $\gamma(t)$ be a complete geodesic in $M$ with $\gamma(0)=p$, parametrized by arclength. By Lemma 2.3 the composition curve $(f \circ \gamma)(t)$ is a Frenet curve in $\bar{M}^{n}(c)$. Denote by $\kappa_{1}(t), \cdots, \kappa_{\ell-1}(t)$ the Frenet curvature functions of $(f \circ \gamma)(t)$, and by $\left\{V_{j}(t) ; 1 \leqq j \leqq \ell\right\}$ the Frenet $\ell$-frame of $(f \circ \gamma)(t)$. Then the parallelity of $f$ means that $V_{j}=V_{j}(0) \in O_{p}^{1}(M)=T_{p}(N)$ for $j=1, \cdots, \ell$. Let $c(t)$ be the Frenet curve in $N$ of osculating rank $\ell$ with the curvature functions $\kappa_{1}(t), \cdots, \kappa_{\ell-1}(t)$ and the initial conditions: $c(0)=p, V_{1}, \cdots, V_{\ell}$. Since $N$ is totally geodesic in $\bar{M}^{n}(c)$, the curve $c(t)$ is a Frenet curve in $\bar{M}^{n}(c)$ with the same curvature functions and initial conditions. Hence we have $(f \circ \gamma)(t)=c(t)$ by the uniqueness of Frenet curves. This means that $f(M) \subset$ $N$. The claim that $O_{q}^{1}(M)=T_{q}(N)$ is obvious by the parallelity of $f$.

The second claims (a), (b), (c) are almost trivial by Lemma 2.1. To complete our proof we may show that $\operatorname{dim}_{C} O_{p}^{1}(M)=\operatorname{dim} M$ in the case 
(c) of Lemma 2.1. Put $N_{p}^{\perp}(M)=\left\{\sigma_{f}^{\perp}(X, Y) ; X, Y \in T_{p}(M)\right\}_{R}$. Then we have $N_{p}^{\perp}(M)=\left\{J T_{p}(M)\right\}^{\perp}$. Since the subspace $O_{p}^{1}(M)$ is $J$-invariant, the subspace $N_{p}^{\perp}(M)$ is also $J$-invariant. Hence, by Lemma 2.2, (3), we have $\sigma_{f}^{\frac{1}{f}}=$ 0 , and thus $O_{p}^{1}(M)=T_{p}(M) \oplus J T_{p}(M)$. This implies that $\operatorname{dim}_{C} O_{p}^{1}(M)=$ $\operatorname{dim} M$.

q.e.d.

Remark 2.5. Let $M$ be a simply connected riemannian symmetric space and $f$ a parallel isometric immersion of $M$ into $\bar{M}^{n}(c), c \neq 0$. As described in Introduction, such $f$ 's have completely classified when they are of type (a) or of type (b) $(c<0)$. Moreover, by the argument of Ferus [4], $f$ of type (b) $(c>0)$ is an equivariant immersion of $M$ into $\boldsymbol{R} \boldsymbol{P}^{s}(c / 4)$. Hence the classification of such $f$ 's is reduced to that of parallel isometric immersions of $M$ into the Euclidean sphere $S^{s}(c / 4)$ of constant sectional curvature $c / 4$ (Ferus [3] for the classification).

\section{§ 3. Parallel submanifolds of type (c), $c>0$}

A complex space form $\bar{M}^{r}(c), c>0$, is the complex projective space $C P^{r}(c)$ of constant holomorphic sectional curvature $c$. Let $D_{i}$ be an $\left(r_{i}+1\right)$ dimensional irreducible symmetric bounded domain of tube type and $D_{i}$ $\longrightarrow C^{r_{i}+1}$ the Harish-Chandra imbedding. Then the Shilov boundary $S_{i}$ $\subset \partial D_{i}$ is an $\left(r_{i}+1\right)$-dimensional compact submanifold in $C^{r_{i+1}}$. The space $\boldsymbol{C}^{r_{i}+1}$ has the canonical hermitian inner product: $C(z, w)=\sum_{j=0}^{r_{i}} z_{j} \bar{w}_{j}$ for $z=\left(z_{j}\right), w=\left(w_{j}\right) \in C^{r_{i}+1}$, and thus the positive definite inner product: $\langle z, w\rangle_{C}=\operatorname{Re} C(z, w)$. The flat riemannian metric on $C^{r_{i+1}}$ induced from the inner product \langle\rangle$_{C}$ is also denoted by \langle\rangle$_{C}$. Let $c_{i}$ be a positive number and $S^{2 r_{i}+1}\left(c_{i}\right)$ the hypersphere of radius $1 / \sqrt{c_{i}}$ with the center 0 . Since $S_{i}$ is contained in some hypersphere, there exists a real number $a_{i}$ such that $a_{i} \cdot S_{i} \subset S^{2 r_{i}+1}\left(c_{i}\right)$. Put $\hat{M}_{i}=a_{i} \cdot S_{i}$. Then the submanifold $\hat{M}_{i}$ $\subset S^{2 r_{i}+1}\left(c_{i}\right)$ is a symmetric $R$-space. For symmetric bounded domains and symmetric $R$-spaces, we refer readers to [16], [18].

Now we have completely classified $r$-dimensional complete totally real parallel submanifolds of $C P^{r}(c)$ (Naitoh-Takeuchi [12]). The classification is performed as follows. For an object $\mathscr{D}=\left(D_{1}, \cdots, D_{s} ; c_{1}, \cdots, c_{s}\right), s \geqq 1$, such that

$$
\sum_{i=1}^{s} \operatorname{dim}_{C} D_{i}=r+1, \quad \sum_{i=1}^{s} 1 / c_{i}=1 / c,
$$

we define a compact submanifold $\hat{M}_{\mathscr{D}}$ by 


$$
\begin{aligned}
\hat{M}_{\mathscr{D}}=\hat{M}_{1} \times \cdots \times \hat{M}_{s} \subset S^{2 r_{1}+1}\left(c_{1} / 4\right) \times \cdots \times S^{2 r_{s}+1}\left(c_{s} / 4\right) \\
\subset S^{2 r+1}(c / 4) \subset C^{r+1} .
\end{aligned}
$$

Then the submanifold $\hat{M}_{\mathscr{D}}$ is totally real in $C^{r+1}$. The riemannian submersion $\pi: S^{2 r+1}(c / 4) \rightarrow C P^{r}(c)$, which is called the Hopf fibring, is a principal $S^{1}$-bundle. For a point $z \in S^{2 r+1}(c / 4)$, the horizontal space $H_{z}$ is invariant by the complex structure $i$ of $C^{r+1}$. Then $i \mid H_{z}$ is compatible with the complex structure $J$ on $T_{\pi(z)}\left(C P^{r}(c)\right)$ by the differential $\pi_{* z}$. Since $\hat{M}_{\mathscr{D}}$ is invariant under the $S^{1}$-action, $M_{\mathscr{D}}=\pi\left(\hat{M}_{\mathscr{g}}\right)$ is an $r$-dimensional compact totally real submanifold of $C P^{r}(c)$.

TheOREM 3.1 (Naitoh-Takeuchi [12]). (1) For an object $\mathscr{D}=\left(D_{1}, \cdots\right.$, $D_{s} ; c_{1}, \cdots, c_{s}$ ) satisfying (3.1), the r-dimensional compact totally real submanifold $M_{\mathscr{D}}$ is parallel.

(2) An r-dimensional complete totally real parallel submanifold of $C P^{r}(c)$ is congruent to some $M_{\mathscr{D}}$.

(3) For objects $\mathscr{D}=\left(D_{1}, \cdots, D_{s} ; c_{1}, \cdots, c_{s}\right), \mathscr{D}^{\prime}=\left(D_{1}^{\prime}, \cdots, D_{t}^{\prime} ; c_{1}^{\prime}, \cdots, c_{t}^{\prime}\right)$ satisfying (3.1), the submanifolds $M_{\mathscr{g}}, M_{\mathscr{I}^{\prime}}$ are congruent to each other if and only if $s=t$ and there exists a permutation $\tau$ such that $D_{\tau(j)}^{\prime}=D_{j}$, $c_{\tau(j)}^{\prime}=c_{j}$ for all $j$.

Suppose that an $r$-dimensional complete totally real parallel submanifold $M$ is not of type (c). Then, by Theorem 2.4, $M$ is totally geodesic. This submanifold $M$ is realized by the object $\mathscr{D}_{0}=\left(D_{I V_{r+1}} ; c\right)$ where $D_{I V_{r+1}}$ is the irreducible symmetric bounded domain of type $\mathrm{IV}_{r+1}$.

Corollary 3.2. All the r-dimensional complete totally real parallel submanifolds of type (c) are realized by objects $\mathscr{D}$ satisfying (3.1) except $\mathscr{D}_{0}$.

Remark 3.3. The classification of totally real parallel isometric immersions of $r$-dimensional simply connected riemannian symmetric spaces into $C P^{r}(c)$ is reduced to that of $r$-dimensional complete totally real parallel submanifolds of $C P^{r}(c)$ (cf. [11], [12]).

An isometric immersion $f$ of a riemannian manifold $M$ into a riemannian manifold $\bar{M}$ is called $\lambda$-isotropic if $\left|\sigma_{f}(X, X)\right|=\lambda$ for a unit vector $X$ of $M$, where $|\zeta|$ denotes the length of a normal vector $\zeta$. A 0 -isotropic immersion is totally geodesic. Nonzero isotropic parallel immersions $f$ into $C P^{n}(c)$ have been studied in [10]. Nonzero isotropic parallel immersions $f$ into $C P^{n}(c)$ of type (a) or (b) have been completely classified in [10], [15]. 
Let $L^{r} \subset C P^{r}(c)$ be an $r(\geqq 2)$-dimensional $\lambda$-isotropic complete parallel submanifold of type (c). Then we have shown that the universal riemannian covering of $L^{r}$ is isometric to one of riemannian symmetric spaces $\boldsymbol{R}^{2}, \boldsymbol{R} \times S^{\ell}(\ell \geqq 2), \quad S U(3) / S O(3), \quad S U(3), \quad S U(6) / S p(3), E_{6} / F_{4}$ and that $\lambda=$ $\sqrt{c} / 2 \sqrt{2}$ ([10, Theorem 4.13]). Moreover we have constructed submanifolds $L^{r}$ concretely and uniquely when universal coverings are $R^{2}$ or $R \times S^{\ell}(\ell \geqq 2)$ ([10, Theorem 6.5]), and have given examples of $L^{r}$ concretely when universal coverings are the other riemannian symmetric spaces ([11, Remark 5.4]). Let $M^{r}$ be an $r(\geqq 2)$-dimensional simply connected riemannian symmetric space and $f: M^{r} \rightarrow C P^{n}(c)$ be a $\lambda$-isotropic parallel isometric immersion of type (c). We may assume that $n=r$ by Theorem 2.4. Then the image $f(M)$ is a parallel submanifold of type (c) and $f: M^{r} \rightarrow f(M)$ is a universal riemannian covering (cf. [11], [12]). Hence, together with Remark 3.3, we have the following

Corollary 3.4. Let $M^{r}$ be an $r(\geqq 2)$-dimensional simply connected riemannian symmetric space and $f: M^{r} \rightarrow C P^{r}(c)$ a $\lambda$-isotropic parallel immersion of type (c). Then $M^{r}$ is isometric to one of the following spaces:

$$
\boldsymbol{R}^{2}, \boldsymbol{R} \times S^{n}(n \geqq 2), S U(3) / S O(3), S U(3), S U(6) / S p(3), E_{6} / F_{4} .
$$

Moreover, the immersions are rigid and $\lambda=\sqrt{c} / 2 \sqrt{2}$.

Remark 3.5. Denote by $D_{\mathrm{I}_{m, m}}, D_{\mathrm{II}_{2 m}}, D_{\mathrm{III}_{m}}, D_{\mathrm{IV}_{m}}, D_{E}$ the irreducible symmetric bounded domains of tube type corresponding to $\mathrm{I}_{m, m}, \mathrm{II}_{2 m}, \mathrm{III}_{m}$, $\mathrm{IV}_{m}$, exceptional types respectively. Then, the object $\mathscr{D}$ corresponding to the submanifold $f(M)$ is $\left(D_{\mathrm{I}_{1,1}}, D_{\mathrm{I}_{1,1}}, D_{\mathrm{I}_{1,1}} ; 3 c, 3 c, 3 c\right),\left(D_{\mathrm{I}_{1,1}}, D_{\mathrm{IV}_{n+1}} ;(n+2) c\right.$, $(n+2) c /(n+1)),\left(D_{\mathrm{III}_{3}} ; c\right),\left(D_{\mathrm{II}_{6}} ; c\right),\left(D_{\mathrm{I}_{3,3}} ; c\right),\left(D_{E} ; c\right)$ according as $M=R^{2}$, $\boldsymbol{R} \times S^{n}(n \geqq 2), S U(3) / S O(3), S U(6) / S p(3), S U(3), E_{6} / F_{4}$ respectively.

\section{§4. Complete inverses of parallel submanifolds of type (c)}

A complex space form $\bar{M}^{r}(c), c<0$, is the complex hyperbolic space $C H^{r}(c)$ of constant holomorphic sectional curvature $c$. We recall fundamental properties for $C H^{r}(c)$. Let $F$ be a hermitian form on the complex vector space $C^{r+1}$ defined by

$$
F(z, w)=-z_{0} \bar{w}_{0}+\sum_{j=1}^{r} z_{j} \bar{w}_{j}
$$

for $z=\left(z_{j}\right), w=\left(w_{j}\right) \in C^{r+1}$. It induces a non-degenerate symmetric bilinear form $\langle z, w\rangle_{F}=\operatorname{Re} F(z, w)$ on $C^{r+1}$. We also denote by \langle\rangle$_{F}$ the flat 
pseudo-riemannian metric on $C^{r+1}$ of signature $(2,2 r)$ induced from \langle\rangle$_{F}$, and by $F^{r+1}$ the flat pseudo-riemannian manifold $\left(C^{r+1},\langle\rangle_{F}\right)$. (We denote by $C^{r+1}$ the flat riemannian manifold $\left(C^{r+1},\langle\rangle_{C}\right)$.) Let $H^{2 r+1}(c / 4)$ be a real hypersurface in $\boldsymbol{F}^{r+1}$ defined by $H^{2 r+1}(c / 4)=\left\{\boldsymbol{z} \in \boldsymbol{F}^{r+1} ; F(z, z)=4 / c\right\}$. The hypersurface $H^{2 r+1}(c / 4)$, with the metric induced from \langle\rangle$_{F}$, is a pseudo-riemannian manifold of signature $(1,2 r)$. Let $\boldsymbol{C H}^{r}$ be the base manifold of the principal $S^{1}$-bundle $H^{2 r+1}(c / 4)$ with the action: $z \rightarrow e^{i \theta} z$. Identify tangent spaces $T_{z}\left(H^{2 r+1}(c / 4)\right), z \in H^{2 r+1}(c / 4)$, with spaces $\left\{w \in F^{r+1}\right.$; $\left.\langle w, z\rangle_{F}=0\right\}$ and subspaces $H_{z}\left(H^{2 r+1}(c / 4)\right) \subset T_{z}\left(H^{2 r+1}(c / 4)\right)$ with subspaces $\left\{w \in F^{r+1} ;\langle w, z\rangle_{F}=\langle w, i z\rangle_{F}=0\right\}$. The restriction of \langle\rangle$_{F}$ into $H_{z}\left(H^{2 r+1}(c / 4)\right)$ is positive definite and the restriction $\pi_{*} \mid H_{z}\left(H^{2 r+1}(c / 4)\right)$ is a linear isomorphism onto $T_{\pi(z)}\left(\mathrm{CH}^{r}\right)$ where $\pi$ denotes the projection of $H^{2 r+1}(c / 4)$ onto $C H^{r}$. Since the complex structure $w \rightarrow i w, w \in H_{z}\left(H^{2 r+1}(c / 4)\right)$, and the restriction \langle\rangle$_{F} \mid H_{z}\left(H^{2 r+1}(c / 4)\right)$ are compatible with the $S^{1}$-action, the linear isomorphism induces an almost complex structure $J$ and a riemannian metric $\left\langle>\right.$ on $\mathrm{CH}^{r}$ such that

$$
\pi_{*}(i X)=J\left(\pi_{*} X\right), \quad\left\langle\pi_{*} X, \pi_{*} Y\right\rangle=\langle X, Y\rangle_{F}
$$

for $X, Y \in H_{z}\left(H^{2 r+1}(c / 4)\right)$. Then we can see that $C H^{r}$ is a Kählerian manifold of constant holomorphic sectional curvature $c$. We denote by $C^{r}(c)$ the Kählerian manifold.

From now on notations $E^{r+1},\langle\rangle_{E}, N^{2 r+1}(c / 4), \pi: N^{2 r+1}(c / 4) \rightarrow \bar{M}^{r}(c)$ denote $\boldsymbol{F}^{r+1},\langle\rangle_{F}, H^{2 r+1}(c / 4), \pi: H^{2 r+1}(c / 4) \rightarrow C H^{r}(c)$, or $\boldsymbol{C}^{r+1},\langle\rangle_{C}$, $S^{2 r+1}(c / 4), \pi: S^{2 r+1}(c / 4) \rightarrow C P^{r}(c)$ according as $c<0$ or $c>0$ respectively. The inclusion $\iota: N^{2 r+1}(c / 4) \rightarrow \boldsymbol{E}^{r+1}$ is totally umbilical, i.e.,

$$
\sigma_{\iota}(X, Y)=-(c / 4)\langle X, Y\rangle_{E} \cdot z
$$

for $X, Y \in T_{z}\left(N^{2 r+1}(c / 4)\right)$ and the mean curvature vector field $\eta_{t}(z)=-(c / 4) \cdot z$, $z \in N^{2 r+1}(c / 4)$, is parallel.

Let $N$ and $B$ be pseudo-riemannian manifolds. A submersion $\tau: N \rightarrow$ $B$ is said to be pseudo-riemannian if, for $p \in N$, the restriction of the metric into the vertical space $V_{p}(N)$ is non-degenerate and the restriction of $\tau_{*}$ into the orthogonal complement $H_{p}(N)$ is an isometry onto $T_{\tau(p)}(B)$. Here $V(N)=\cup_{p \in N} V_{p}(N)$ (resp. $H(N)=\cup_{p \in N} H_{p}(N)$ ) is called the vertical (resp. the horizontal) subbundle of $T(N)$. For a vector field $X$ of $N$, its $V(N)$ component and $H(N)$-component will be denoted by $\mathscr{V} X$ and $\mathscr{H} X$ respectively. If $\mathscr{V} X=X$ (resp. $\mathscr{H} X=X), X$ is said to be vertical (resp. horizontal). 
If $X$ is horizontal and projectable to a vector field $X_{*}$ of $B$, it is called the horizontal lift of $X_{*}$ and denoted by $X=$ h.l. $X_{*}$. If $N, B$ are riemannian manifolds, a pseudo-riemannian submersion is riemannian in the sense of O'Neill [14]. He defined the fundamental tensors $A, T$ for a riemannian submersion. We can also define them for a pseudo-riemannian submersion in the same way as for a riemannian submersion. (See O'Neill [14] for the definition of $A, T$.) The submersion $\pi: N^{2 r+1}(c / 4) \rightarrow \bar{M}^{r}(c)$ is pseudo-riemannian with horizontal subspaces

$$
H_{z}\left(N^{2 r+1}(c / 4)\right)=\left\{w \in T_{z}\left(N^{2 r+1}(c / 4)\right) ;\langle w, z\rangle_{E}=\langle w, i z\rangle_{E}=0\right\} .
$$

The Levi-Civita connections of $N^{2 r+1}(c / 4), \bar{M}^{r}(c)$ are denoted by $\nabla^{N}$, $\bar{\nabla}$ respectively. Let $\nu$ be a normal vector field defined by $\nu_{z}=(\sqrt{|c|} / 2) z$, $z \in N^{2 r+1}(c / 4)$. Then we have $\left\langle\nu_{z}, \nu_{z}\right\rangle_{E}=-1,1$ according as $c<0, c>0$. Since each fibre of $\pi$ is a geodesic in $N^{2 r+1}(c / 4)$, the fundamental tensor $T$ equals zero. Then we have the following identities:

$$
\nabla_{V}^{N} X=\mathscr{H} \nabla_{V}^{N} X, \quad \nabla_{X}^{N} V=A_{X} V+\mathscr{V} \nabla_{X}^{N} V, \quad \nabla_{X}^{N} Y=\mathscr{H} \nabla_{X}^{N} Y+A_{X} Y
$$

for horizontal vector fields $X, Y$ and a vertical vector field $V$, and

$$
\mathscr{H} \nabla_{V}^{N} X=A_{X} V, \quad \mathscr{H} \nabla_{X}^{N} Y=h \cdot \ell \cdot \bar{\nabla}_{X_{*}} Y_{*}
$$

for $X=$ h.l. $X_{*}, Y=$ h.l. $Y_{*}$. The fundamental tensor $A$ for $\pi$ is given by

$$
\left\{\begin{aligned}
A_{X} i \nu & =(\sqrt{|c|} / 2) i X, \\
A_{X} Y & =\left\{\begin{aligned}
-(\sqrt{|c|} / 2)\langle X, i Y\rangle_{E} i \nu & \text { if } c<0, \\
(\sqrt{|c|} \mid / 2)\langle X, i Y\rangle_{E} i \nu & \text { if } c>0 .
\end{aligned}\right.
\end{aligned}\right.
$$

(See O'Neill [14] for the proof of (4.2) (4.4).)

Let $M^{r}$ be an $r$-dimensional totally real submanifold of $\bar{M}^{r}(c)$ and put $\hat{M}^{r+1}=\pi^{-1}\left(M^{r}\right) \subset N^{2 r+1}(c / 4)$, which is called the complete inverse of $M^{r}$. Set $H_{z}(\hat{M})=T_{z}(\hat{M}) \cap H_{z}\left(N^{2 r+1}(c / 4)\right)$ for $z \in \hat{M}$. Then we have the orthogonal decomposition $T_{z}(\hat{M})=V_{z}\left(N^{2 r+1}(c / 4)\right) \oplus H_{z}(\hat{M})$ for the metric on $\hat{M}$ induced from \langle\rangle$_{E}$. Hence $\hat{M}$ has signature $(1, r),(0, r+1)$ according as $c<0, c>0$ respectively, and $\pi: \hat{M} \rightarrow M$ is a pseudo-riemannian submersion with horizontal subspaces $H_{z}(\hat{M}), z \in \hat{M}$. The total reality of $M$ implies that

$$
\left\langle i H_{z}(\hat{M}), H_{z}(\hat{M})\right\rangle_{E}=\{0\} .
$$

Let $\hat{\nabla}, \nabla$ be the Levi-Civita connections of $\hat{M}, M$ and $\hat{\sigma}, \sigma$ the second 
fundamental forms of $\hat{M} \rightarrow N^{2 r+1}(c / 4), M \rightarrow \bar{M}^{r}(c)$ respectively. Then, by $(4.2) \sim(4.5)$, we have the following identities:

$$
\left\{\begin{array}{l}
\hat{V}_{X} Y=h \cdot \ell \cdot \nabla_{X_{*}} Y_{*}, \quad \hat{\sigma}(X, Y)=h \cdot \ell \cdot \sigma\left(X_{*}, Y_{*}\right), \\
\hat{\nabla}_{X} i \nu=\mathscr{V} \nabla_{X}^{N} i \nu, \quad \hat{\sigma}(X, i \nu)=(\sqrt{|c|} \mid / 2) i X, \\
\hat{\nabla}_{i \nu} X=\hat{V}_{i \nu} i \nu=0, \quad \hat{\sigma}(i \nu, i \nu)=0
\end{array}\right.
$$

for vector fields $X, Y$ of $\hat{M}$ which are horizontal lifts of vector fields $X_{*}$, $Y_{*}$ of $M$ respectively (Lemma 1.1, [12]).

A submanifold $N \subset \boldsymbol{R}^{m}$ is said to be substantial if $N$ is not contained in any affine hyperplane of $\boldsymbol{R}^{m}$.

A pseudo-riemannian manifold is called complete if the Levi-Civita connection is complete. A pseudo-riemannian manifold $N$ is said to be a pseudo-riemannian symmetric space if, for each point $p \in N$, the geodesic symmetry $s_{p}$ at $p$ can be extended to a global isometry of $N$. A pseudoriemannian symmetric space is complete (cf. [7]).

Proposition 4.1. Let $M^{r}$ be an r-dimensional totally real submanifold of $\bar{M}^{r}(c)$ and $\hat{M}^{r+1}$ the complete inverse of $M^{r}$. Then,

(1) $M$ is minimal in $\bar{M}^{r}(c)$ if and only if $\hat{M}$ is minimal in $N^{2 r+1}(c / 4)$,

(2) $V(\hat{M}), H(\hat{M})$ are parallel subbundles of $T(\hat{M})$,

(3) $M$ is parallel in $\bar{M}^{r}(c)$ if and only if $\hat{M}$ is parallel in $N^{2 r+1}(c / 4)$,

(4) $\hat{M}$ is substantial in $\boldsymbol{E}^{r+1}=\boldsymbol{R}^{2 r+2}$.

Assume that $M$ is parallel in $\bar{M}^{r}(c)$ if $c<0$. Then,

(5) $M$ is complete if and only if $\hat{M}$ is complete.

Proof. If $c>0$, our claims are results of Lemma 1.1, [12]. Assume that $c<0$. Claims (1), (2), (3) are proved in the same way as in the case $c>0$. We show claims (4), (5).

(4) Assume that $\hat{M}$ is contained in some real hyperplane of $\boldsymbol{F}^{r+1}$. Then there exists a complex linear hyperspace $V^{r}$ of $F^{r+1}$ which contains $\hat{M}$ (See the proof of Lemma 1.1, (3), [12]). Since $\hat{M}$ is totally real in $F^{r+1}$, we have $T_{z}(\hat{M}) \cap i T_{z}(\hat{M})=\{0\}$ for $z \in \hat{M}$. Identify $T_{z}(V)$ with $V$. Then the complex linear subspace $T_{z}(\hat{M}) \oplus i T_{z}(\hat{M})$ is contained in $V$. This is a contradiction since $\operatorname{dim}_{C}\left\{T_{z}(\hat{M}) \oplus i T_{z}(\hat{M})\right\}=r+1$. Hence $\hat{M}$ is substantial in $\boldsymbol{F}^{r+1}$.

(5) Since $H(\hat{M})$ is a parallel subbundle of $T(\hat{M})$, there exists a totally geodesic maximal integral submanifold $H^{r}$ of $H(\hat{M})$. Then $H^{r}$ is riemannian and $\pi: H^{r} \rightarrow M^{r}$ is an isometric immersion. 
If $\hat{M}$ is complete, so is $H^{r}$ by the maximality. Thus $M^{r}$ is complete. Conversely, assume that $M^{r}$ is complete. Let $\left.《\right\rangle$ be a riemannian metric on $\hat{M}$ constructed from the pseudo-riemannian metric \langle\rangle$_{F}$ as follows: $\left\langle\langle X+V, Y+W\rangle=\langle X, Y\rangle_{F}-\langle V, W\rangle_{F}\right.$ for $X, Y \in H_{z}(\hat{M}), V, W \in V_{z}(\hat{M})$. Then $\pi:(\hat{M}, 《\rangle) \rightarrow M$ is a riemannian submersion with the same horizontal subbundle $H(\hat{M})$ as that of the pseudo-riemannian submersion $\pi$ : $\left(\hat{M},\langle\rangle_{F}\right) \rightarrow M$. Note that $(\hat{M}, 《 \Downarrow)$ is complete by the compactness of fibres. Let $\hat{V}$ be the Levi-Civita connection of $(\hat{M}, 《\rangle)$. If $X$ is a horizontal vector field for $\pi:(\hat{M}, 《 \Downarrow) \rightarrow M$, so is $\widehat{\nabla}_{X} X$ ([14]). This implies that the maximal integral submanifold $H^{r}$ is totally geodesic in $\left.(\hat{M}, 《\rangle\right)$. Hence $H^{r}$ is complete by the maximality. Now define $\phi: S^{1} \times H^{r} \rightarrow \hat{M}$ by $\phi\left(e^{i \theta}, z\right)=e^{i \theta} \cdot z$ for $e^{i \theta} \in S^{1}, z \in H^{r}$. Then $\phi$ is a covering map. In fact, let \langle\rangle$_{S_{1}}$ be a riemannian metric on $S^{1}$ such that $\left\langle i e^{i \theta}, i e^{i \theta}\right\rangle_{S^{1}}=-4 / c$ for any $\theta$. Then $\phi$ is isometric immersion of the complete riemannian manifold $\left(S^{1},\langle\rangle_{S^{1}}\right) \times\left(H^{r},\langle\rangle_{F}\right)$ into $\left.(\hat{M}, 《\rangle\right)$. Thus $\phi$ is a covering map. Let $\left(\tilde{H}^{r},\langle\rangle_{F}\right)$ be the universal riemannian covering of $\left(H^{r},\langle\rangle_{F}\right)$. Since $\left(H^{r},\langle\rangle_{F}\right)$ is a complete riemannian locally symmetric space, $\left(\tilde{H}^{r},\langle\sim\rangle_{F}\right)$ is a riemannian symmetric space. Thus $\left(S^{1},-\langle\rangle_{S^{1}}\right) \times\left(\tilde{H}^{r},\left\langle^{\sim}\right\rangle_{F}\right)$ is a pseudo-riemannian symmetric space. This implies that the space is complete. Hence $\left(\hat{M},\langle\rangle_{F}\right)$ is complete through the pseudo-riemannian covering $\left(S^{1},-\langle\rangle_{S 1}\right) \times\left(\tilde{H}^{r},\left\langle^{\sim}\right\rangle_{F}\right) \rightarrow\left(S^{1},-\langle\rangle_{S 1}\right) \times\left(H^{r},\langle\rangle_{F}\right) \rightarrow\left(\hat{M},\langle\rangle_{F}\right)$. q.e.d.

Let $M$ be a complete parallel submanifold of a riemannian symmetric space. Then $M$ is a complete riemannian locally symmetric space and thus the universal riemannian covering space of $M$ is a simply connected riemannian symmetric space.

Proposition 4.2. Let $M^{r}$ be an $r$-dimensional complete totally real parallel submanifold of $\bar{M}^{r}(c)$. Then irreducible factors of the universal covering space of $M^{r}$ are isometric to some of the following riemannian symmetric spaces:

$$
\begin{aligned}
& S O(1, k) / S O(k), \quad S O(k+1) / S O(k)(k \geqq 2), \quad S U(\ell) / S O(\ell), \quad S U(\ell), \\
& S U(2 \ell) / S p(\ell)(\ell \geqq 3), \quad E_{6} / F_{4} .
\end{aligned}
$$

Proof. Let $N$ be a riemannian symmetric space. Fix a point $p \in N$ and denote by $S^{3}\left(T_{p}(N)\right)$ the vector space of symmetric trilinear forms on $T_{p}(N)$. Identify elements of $S^{3}(T(N))$ with $T_{p}(N)$-valued bilinear forms 
on $T_{p}(N)$ through the metric $\left\langle>\right.$. For $\tau \in S^{3}\left(T_{p}(N)\right), X \in T_{p}(N)$, define a symmetric endomorphism $\tau(X)$ of $T_{p}(N)$ by $\tau(X) Y=\tau(X, Y)$ for $Y \in T_{p}(N)$. Let $R^{N}, \mathfrak{f}^{N}$ be the curvature tensor, the holonomy algebra of $N$ respectively. Put

$$
\mathscr{M}_{N}=\left\{\tau \in S^{3}\left(T_{p}(N)\right) ; \mathfrak{f}^{N} \cdot \tau=0\right\}
$$

and, for $d \in \boldsymbol{R}$,

$$
\mathscr{M}_{N}(d)=\left\{\tau \in \mathscr{M}_{N} ; \begin{array}{l}
d(\langle Y, Z\rangle X-\langle X, Z\rangle Y)=R^{N}(X, Y) Z-[\tau(X), \tau(Y)] Z \\
\text { for } X, Y, Z \in T_{p}(N)
\end{array}\right\}
$$

Assume that $N$ is irreducible. Then $\operatorname{dim} \mathscr{M}_{N}=1$ if $N$ is one of the following spaces and their non-compact duals, and $\operatorname{dim} \mathscr{M}_{N}=0$ otherwise:

$$
S U(\ell) / S O(\ell), \quad S U(\ell), \quad S U(2 \ell) / S p(\ell)(\ell \geqq 3), \quad E_{6} / F_{4}
$$

(Naitoh [11], Lemma 4.2).

Let $\tilde{M}$ be the universal riemannian covering space of $M$ and $N$ an irreducible factor of $\tilde{M}$. Denote by $\tilde{\sigma}$ the second fundamental form of the isometric immersion $\tilde{M} \rightarrow \bar{M}^{r}(c)$. Define an element $\tilde{\sigma}_{N} \in S^{3}\left(T_{p}(N)\right)$ by

$$
\left\langle\tilde{\sigma}_{N}(X, Y), Z\right\rangle=\langle J \tilde{\sigma}(X, Y), Z\rangle
$$

for $X, Y, Z \in T_{p}(N)$. Then there exists a real number $s$ such that $\mathscr{M}_{N}(s)$ $\ni \tilde{\sigma}_{N}$ (Naitoh [11], Theorem 6.4, (A), (2)). If $N$ is none of the spaces in (4.7) and their non-compact duals, $\mathscr{M}_{N}(s)=\{0\}$, i.e., $\tilde{\sigma}_{N}=0$ since $\operatorname{dim} \mathscr{M}_{N}$ $=0$. Hence we have

$$
R^{N}(X, Y) Z=s(\langle Y, Z\rangle X-\langle X, Z\rangle Y)
$$

for $X, Y, Z \in T_{p}(N)$. This implies that $N$ has constant sectional curvature. Since $N$ is irreducible, it is not flat, and thus is one of $S O(1, k)$ / $S O(k), S O(k+1) / S O(k)(k \geqq 2)$.

Let $N^{*}$ be one of the spaces in (4.7) and assume that $N$ is the noncompact dual of $N^{*}$. Identify the tangent space $T_{p}(N)$ with a tangent space $T_{p^{*}}\left(N^{*}\right)$ by the duality. Then the curvature tensor $R^{N^{*}}$ at $p^{*}$ is identified with $-R^{N}$ at $p$, and thus the representation $\left(\mathfrak{f}_{N^{*}}, T_{p^{*}}\left(N^{*}\right)\right)$ is compatible with $\left(\mathfrak{f}_{N}, T_{p}(N)\right)$ (See Helgason [6] for the duality). Since $N^{*}$ is of compact type, there exists a number $d>0$ such that $\mathscr{M}_{N^{*}}(d)=\{\lambda \neq 0\}$ (Naitoh [11], Proposition 4.4). Identify $\mathscr{M}_{N N^{*}}$ with $\mathscr{M}_{N}$. Since $\operatorname{dim} \mathscr{M}_{N}=1$, there exists $\alpha \in \boldsymbol{R}$ such that $\tilde{\sigma}_{N}=\alpha \lambda$. Then we have 


$$
\begin{aligned}
s(\langle Y, Z\rangle X-\langle X, Z\rangle Y) & =R^{N}(X, Y) Z-\left[\tilde{\sigma}_{N}(X), \tilde{\sigma}_{N}(Y)\right] Z \\
& =R^{N}(X, Y) Z-\alpha^{2}[\lambda(X), \lambda(Y)] Z
\end{aligned}
$$

and

$$
d(\langle Y, Z\rangle X-\langle X, Z\rangle Y)=-R^{v}(X, Y) Z-[\lambda(X), \lambda(Y)] Z
$$

for $X, Y, Z \in T_{p}(N)$, and thus

$$
R^{N}(X, Y) Z=\left(s-\alpha^{2} d\right) /\left(\alpha^{2}+1\right)(\langle Y, Z\rangle X-\langle X, Z\rangle Y) .
$$

This implies that $N$ has constant sectional curvature, which is a contradiction.

Hence $N$ is one of $S O(1, k) / S O(k), S O(k+1) / S O(k)(k \geqq 2)$, and the spaces in (4.7).

q.e.d.

\section{§5. Jordan triple systems and symmetric graded Lie algebras}

In this section we recall Jordan triple systems and symmetric graded Lie algebras, and define "orthogonal" Jordan triple systems and "orthogonal" symmetric graded Lie algebras. These notions play important roles for the classification of totally real parallel submanifolds of $\bar{M}^{r}(c), c \neq 0$, of type (c). The classification will be attained in the second series of this paper.

Let $V$ be a finite dimensional real vector space and $\{,$,$\} a V$-valued trilinear form on $V$. Define endomorphisms $L(X, Y), X, Y \in V$, by $L(X, Y) Z$ $=\{X, Y, Z\}$ for $Z \in V$. An object $(V,\{\})$ is called a Jordan triple system (abbreviated as JTS) if the following two conditions are satisfied:

(JT 1) $L(X, Y) Z=L(Z, Y) X$,

(JT 2) $[L(W, Z), L(X, Y)]=L(L(W, Z) X, Y)-L(X, L(Z, W) Y)$

for $W, Z, X, Y \in V$. The trace form $\beta$ of a $\operatorname{JTS}(V,\{\})$ is a bilinear form on $V$ defined by $\beta(X, Y)=\operatorname{Tr} L(X, Y)$ for $X, Y \in V$. A JTS is called nondegenerate if the trace form is non-degenerate. Then the trace form is always symmetric. It is said that two JTS's $(V,\{\}),\left(V^{\prime},\{\}^{\prime}\right)$ are equivalent to each other if there exists a linear isomorphism $g$ of $V$ onto $V^{\prime}$ such that $g\{X, Y, Z\}=\{g(X), g(Y), g(Z)\}^{\prime}$ for $X, Y, Z \in V$.

For a JTS $(V,\{\})$ we denote by $\mathscr{S}(V,\{\})$ the set of non-degenerate symmetric bilinear forms $\langle$,$\rangle on V$ such that $L(X, Y)^{t}=L(Y, X)$ for $X$, $Y \in V$, where $L(X, Y)^{t}$ denotes the transpose endomorphism of $L(X, Y)$ for \langle\rangle . It is known that the trace form $\beta$ belongs to $\mathscr{S}(V,\{\})$ for a non- 
degenerate $\operatorname{JTS}(V,\{\})$. Assume that $\mathscr{S}(V,\{\}) \neq \phi$. An object $(V,\{\}$; \langle\rangle ) is called an orthogonal Jordan triple system (abbreviated as OJTS) for a JTS $(V,\{\})$ and a form $\langle>\in \mathscr{P}(V,\{\})$. It is said that two OJTS's $\left(V,\{\quad\} ;\langle>),\left(V^{\prime},\{\}^{\prime} ;\langle\rangle^{\prime}\right)\right.$ are equivalent to each other if there exists a linear isomorphism $g$ of $V$ onto $V^{\prime}$ such that $g\{X, Y, Z\}=\{g(X), g(Y)$, $g(Z)\}^{\prime},\langle g(X), g(Y)\rangle^{\prime}=\langle X, Y\rangle$ for $X, Y \in V$.

A symmetric Lie algebra $(\mathfrak{g}, \rho)$ (abbreviated as SLA $(\mathfrak{g}, \rho)$ ) is a pair of a finite dimensional real Lie algebra $g$ and an involutive automorphism $\rho$ of $g$ such that $\rho \neq \mathrm{id}_{\mathfrak{g}}$. Let

$$
\mathfrak{l}=\{X \in \mathfrak{g} ; \rho(X)=X\}, \quad \mathfrak{p}=\{X \in \mathfrak{g} ; \rho(X)=-X\} .
$$

An SLA $(\mathfrak{g}, \rho)$ is called effective if the representation $\left(\left.\operatorname{ad}(\mathfrak{f})\right|_{\mathfrak{p}}, \mathfrak{p}\right)$ of $\mathfrak{f}$ is faithful.

For an SLA $(\mathfrak{g}, \rho)$ we denote by $\mathscr{S}(\mathfrak{g}, \rho)$ the set of non-degenerate symmetric bilinear forms \langle\rangle$_{\mathfrak{p}}$ on $\mathfrak{p}$ such that ad $\left.(T)\right|_{\mathfrak{p}}, T \in \mathfrak{f}$, are skew symmetric for \langle\rangle$_{\mathfrak{p}}$. Assume that $\mathscr{S}(\mathfrak{g}, \rho) \neq \phi$. An object $\left(\mathfrak{g}, \rho,\langle\rangle_{\mathfrak{p}}\right)$ is called an orthogonal symmetric Lie algebra (abbreviated as OSLA) for an SLA $(\mathfrak{g}, \rho)$ and a form \langle\rangle$_{\mathfrak{p}} \in \mathscr{S}(\mathfrak{g}, \rho)$.

A complex symmetric Lie algebra ( $\mathfrak{g}, \rho, J_{\mathfrak{p}}$ ) (abbreviated as CSLA ( $\mathfrak{g}, \rho$, $\left.J_{\mathfrak{p}}\right)$ ) is a pair of an $\operatorname{SLA}(\mathfrak{g}, \rho)$ and an almost complex structure $J_{\mathfrak{p}}$ on $\mathfrak{p}$ such that $\left.\operatorname{ad}(T)\right|_{\mathfrak{p}} \circ J_{\mathfrak{p}}=J_{\mathfrak{p}} \circ$ ad $\left.(T)\right|_{\mathfrak{p}}$ for $T \in \mathfrak{f}$. For a CSLA $\left(\mathfrak{g}, \rho, J_{\mathfrak{p}}\right)$ we denote by $\mathscr{S}\left(\mathfrak{g}, \rho, J_{\mathfrak{p}}\right)$ the set of forms \langle\rangle$_{\mathfrak{p}} \in \mathscr{S}(\mathfrak{g}, \rho)$ such that $\left\langle J_{\mathfrak{p}} X, J_{p} Y\right\rangle_{\mathfrak{p}}$ $=\langle X, Y\rangle_{\mathfrak{p}}$ for $X, Y \in \mathfrak{p}$. Assume that $\mathscr{S}\left(\mathfrak{g}, \rho, J_{\mathfrak{p}}\right) \neq \phi$. An object $\left(\mathfrak{g}, \rho, J_{\mathfrak{p}}\right.$, \langle\rangle$_{\mathfrak{p}}$ ) is called a Hermitian symmetric Lie algebra (abbreviated as HSLA) for a $\operatorname{CSLA}\left(\mathfrak{g}, \rho, J_{\mathfrak{p}}\right)$ and a form \langle\rangle$_{\mathfrak{p}} \in \mathscr{S}\left(\mathfrak{g}, \rho, J_{\mathfrak{p}}\right)$.

An SLA $(\mathfrak{g}, \rho)$ is called a symmetric graded Lie algebra (abbreviated as SGLA) if the following four conditions are satisfied:

(SGL 1) $\mathfrak{g}=\mathfrak{g}_{-1}+\mathfrak{g}_{0}+\mathfrak{g}_{1}$ is a graded Lie algebra, i.e., $\left[\mathfrak{g}_{\mu}, \mathfrak{g}_{\nu}\right.$ ] $\subset \mathfrak{g}_{\mu+\nu}$ for $\mu, \nu \in Z$, where $g_{\lambda}=\{0\}$ for $\lambda \neq 0, \pm 1$.

(SGL 2) $\rho\left(\mathfrak{g}_{\mu}\right)=\mathfrak{g}_{-\mu}$ for $\mu=0, \pm 1$.

(SGL 3) $g_{0}$ acts faithfully on $\mathfrak{g}_{-1} \neq\{0\}$.

(SGL 4) $\mathfrak{g}_{0}=\left[\mathfrak{g}_{-1}, \mathfrak{g}_{1}\right]$.

An SGLA ( $\left.\mathfrak{g}=\sum \mathfrak{g}_{\mu}, \rho\right)$ is called semi-simple if $\mathfrak{g}$ is semi-simple. For a semi-simple SGLA the condition (SGL 4) is automatically attained from other conditions. It is said that two SGLA's $\left(\mathfrak{g}=\sum \mathfrak{g}_{\mu}, \rho\right),\left(\mathfrak{g}^{\prime}=\sum \mathfrak{g}_{\mu}^{\prime}, \rho^{\prime}\right)$ are equivalent to each other if there exists a Lie algebra isomorphism $\tau$ of $\mathfrak{g}$ onto $\mathfrak{g}^{\prime}$ such that $\tau\left(\mathfrak{g}_{\mu}\right)=\mathfrak{g}_{\mu}^{\prime}$ for $\mu=0, \pm 1$ and $\tau \circ \rho=\rho^{\prime} \circ \tau$. Here $\tau$ is 
called a symmetric graded Lie algebra isomorphism, abbreviated as SGLAisomorphism.

Let $\left(\mathfrak{g}=\sum \mathfrak{g}_{\mu}, \rho\right)$ be an SGLA. Then an OSLA $\left(\mathfrak{g}, \rho,\langle\rangle_{p}\right)$ (resp. a HSLA $\left.\left(g, \rho, J_{\mathfrak{p}},\langle\rangle_{\mathfrak{p}}\right)\right)$ is called an orthogonal symmetric graded Lie algebra, abbreviated as OSGLA (resp. a Hermitian symmetric graded Lie algebra, abbreviated as HSGLA). It is said that two OSGLA's $\left(\mathfrak{g}=\sum \mathfrak{g}_{\mu}, \rho,\langle\rangle_{\mathrm{p}}\right)$, $\left(\mathfrak{g}^{\prime}=\sum \mathfrak{g}_{\mu}^{\prime}, \rho^{\prime},\langle\rangle_{p^{\prime}}\right)$ (resp. HSGLA's $\left(\mathfrak{g}=\sum \mathfrak{g}_{\mu}, \rho, J_{p},\langle\rangle_{p}\right), \quad\left(\mathfrak{g}^{\prime}=\sum \mathfrak{g}_{\mu}^{\prime}, \rho^{\prime}\right.$, $\left.J_{p^{\prime}},\langle\rangle_{p^{\prime}}\right)$ ) are equivalent to each other if there exists an SGLA-isomorphism $\tau$ of $\left(\mathfrak{g}=\sum \mathfrak{g}_{\mu}, \rho\right)$ onto $\left(\mathfrak{g}^{\prime}=\sum \mathfrak{p}_{\mu}^{\prime}, \rho^{\prime}\right)$ such that $\langle\tau(X), \tau(Y)\rangle_{p^{\prime}}=\langle X$, $Y\rangle_{\mathfrak{p}}$ for $X, Y \in \mathfrak{p}\left(\operatorname{resp} .\langle\tau(X), \tau(Y)\rangle_{\mathfrak{p}^{\prime}}=\langle X, Y\rangle_{\mathfrak{p}}\right.$ for $X, Y \in \mathfrak{p}$ and $\tau \circ J_{\mathfrak{p}}=$ $\left.J_{p^{\prime}} \circ \tau\right)$.

We refer to Satake [16] for non-degenerate JTS's and semi-simple SGLA's.

Now we study a correspondence between OJTS's and OSGLA's. Let $(V,\{\},\langle\rangle)$ be an OJTS. Put $L=\{L(X, Y) ; X, Y \in V\}_{R}, \mathfrak{g}=V+L+V$, $\mathrm{g}_{-1}=V+0+0, \mathrm{~g}_{0}=0+L+0, \mathrm{~g}_{1}=0+0+V$. Define a bracket product $[$, ] on $g$ by

$$
\begin{aligned}
& {[(X, F, Y),(Z, G, W)]} \\
& \quad=(F(Z)-G(X),[F, G]-(1 / 2) L(X, W) \\
& \left.\quad+(1 / 2) L(Z, Y), G^{t}(Y)-F^{t}(W)\right)
\end{aligned}
$$

for $X, Y, Z, W \in V, F, G \in L$, and a linear isomorphism $\rho$ of $\mathrm{g}$ by

$$
\rho(X, F, Y)=\left(Y,-F^{t}, X\right)
$$

for $X, Y \in V, F \in L$.

Lemma 5.1. The object $\left(\mathfrak{g}=\sum \mathfrak{g}_{\mu}, \rho\right)$ is an $S G L A$ and is independent of the choice of $\langle>\in \mathscr{S}(V,\{\})$. Moreover $\mathfrak{f}, \mathfrak{p}$ are given by

$$
\begin{aligned}
& \mathfrak{f}=\{(X, L(Y, Z)-L(Z, Y), X) ; X, Y, Z \in V\}_{R}, \\
& \mathfrak{p}=\{(X, L(Y, Z)+L(Z, Y),-X) ; X, Y, Z \in V\}_{R} .
\end{aligned}
$$

Proof. We show the Jacobi identity for the bracket product [, ]. Note that

$$
\begin{gathered}
L(X, Y)^{t}=L(Y, X) \\
L(X, Y)^{t} Z=L(X, Z)^{t} Y, \\
{[F, L(X, Y)]=L(F(X), Y)-L\left(X, F^{t}(Y)\right)}
\end{gathered}
$$


for $X, Y \in V, F \in L$. In fact, (5.4) is attained by (5.3), (JT 1), and (5.5) is attained by (5.3), (JT 2). Then the Jacobi identity is proved straightforwardly by $(5.3) \sim(5.5)$.

The identity (5.3) also implies that $\rho$ is an involutive automorphism of g. The other claims are obvious.

q.e.d.

We call this $\left(\mathfrak{g}=\sum \mathfrak{g}_{\mu}, \rho\right)$ the SGLA associated with a $\operatorname{JTS}(V,\{\})$ such that $\mathscr{S}(V,\{\}) \neq \phi$.

Let $(V,\{\})$ be a JTS and $\left(\mathfrak{g}=\sum \mathfrak{g}_{\mu}, \rho\right)$ the SGLA associated with $(V,\{\})$. For a form $\langle>\in \mathscr{S}(V,\{\})$ define a symmetric bilinear form \langle\rangle$_{\mathfrak{p}}$ by

$$
\begin{aligned}
\langle(X, F,-X),(Y, G,-Y)\rangle_{\mathfrak{p}} & =\langle X, Y\rangle+\sum_{i=1}^{k}\left\langle G\left(Z_{i}\right), W_{i}\right\rangle \\
& =\langle X, Y\rangle+\sum_{j=1}^{\ell}\left\langle F\left(Z_{j}^{\prime}\right), W_{j}^{\prime}\right\rangle
\end{aligned}
$$

for $(X, F,-X),(Y, G,-Y) \in \mathfrak{p}$, where $2 F=\sum_{i=1}^{k}\left(L\left(Z_{i}, W_{i}\right)+L\left(W_{i}, Z_{i}\right)\right), 2 G$ $=\sum_{j=1}^{\ell}\left(L\left(Z_{j}^{\prime}, W_{j}^{\prime}\right)+L\left(W_{j}^{\prime}, Z_{j}^{\prime}\right)\right)$.

Lemma 5.2. The form \langle\rangle$_{p}$ is a well-defined form in $\mathscr{S}(\mathrm{g}, \rho)$.

Proof. We show that $\sum_{i=1}^{k}\left\langle G\left(Z_{i}\right), W_{i}\right\rangle=\sum_{j=1}^{\ell}\left\langle F\left(Z_{j}^{\prime}\right), W_{j}^{\prime}\right\rangle$. Note that

$$
\begin{aligned}
\langle L(X, Y) Z, W\rangle & =\langle L(Y, X) W, Z\rangle=\langle L(W, X) Y, Z\rangle \\
& =\langle L(X, W) Z, Y\rangle=\langle L(Z, W) X, Y\rangle
\end{aligned}
$$

for $X, Y, Z, W \in V$ by (5.3), (JT 1). Then we have

$$
\begin{aligned}
\sum_{i}\left\langle G\left(Z_{i}\right), W_{i}\right\rangle & =(1 / 2) \sum_{i, j}\left(\left\langle L\left(Z_{j}^{\prime}, W_{j}^{\prime}\right) Z_{i}, W_{i}\right\rangle+\left\langle L\left(W_{j}^{\prime}, Z_{j}^{\prime}\right) Z_{i}, W_{i}\right\rangle\right) \\
& =(1 / 2) \sum_{i, j}\left(\left\langle L\left(Z_{i}, W_{i}\right) Z_{j}^{\prime}, W_{j}^{\prime}\right\rangle+\left\langle L\left(Z_{i}, W_{i}\right) W_{j}^{\prime}, Z_{j}^{\prime}\right\rangle\right) \\
& =(1 / 2) \sum_{i, j}\left(\left\langle L\left(Z_{i}, W_{i}\right) Z_{j}^{\prime}, W_{j}^{\prime}\right\rangle+\left\langle L\left(W_{i}, Z_{i}\right) Z_{j}^{\prime}, W_{j}^{\prime}\right\rangle\right) \\
& =\sum_{j}\left\langle F\left(Z_{j}^{\prime}\right), W_{j}^{\prime}\right\rangle .
\end{aligned}
$$

This implies that \langle\rangle$_{\mathrm{p}}$ is a well-defined symmetric bilinear form on $\mathfrak{p}$.

Assume that $\langle(X, F,-X),(Y, G,-Y)\rangle_{\mathfrak{p}}=0$ for $(Y, G,-Y) \in \mathfrak{p}$. Put $G=0$. Then we have $\langle X, Y\rangle=0$ for $Y \in V$, and thus $X=0$ by the nondegeneracy of \langle\rangle . Moreover, putting $2 G=L(Z, W)+L(W, Z)$, we have $\langle F(Z), W\rangle=0$ for $Z, W \in V$, and thus $F=0$. Hence the form \langle\rangle$_{\mathfrak{p}}$ is non-degenerate.

Finally we show that ad $\left.(T)\right|_{p}, T \in \mathbb{1}$, are skew symmetric for \langle\rangle$_{p}$. Put $T=(0, K, 0) \in \mathfrak{f}, 2 F=L(X, Y)+L(Y, X), 2 F^{\prime}=L\left(X^{\prime}, Y^{\prime}\right)+L\left(Y^{\prime}, X^{\prime}\right)$ $\in \mathfrak{p}$. Then we have 


$$
\begin{aligned}
&\left\langle(0,[K, F], 0),\left(0, F^{\prime}, 0\right)\right\rangle_{\mathfrak{p}}=\left\langle[K, F]\left(X^{\prime}\right), Y^{\prime}\right\rangle \\
&=-\left(\left\langle F\left(X^{\prime}\right), K\left(Y^{\prime}\right)\right\rangle+\left\langle K\left(X^{\prime}\right), F\left(Y^{\prime}\right)\right\rangle\right) \\
&=-\frac{1}{2}\left\langle\left(0, L\left(X^{\prime}, K\left(Y^{\prime}\right)\right)+L\left(K\left(Y^{\prime}\right), X^{\prime}\right), 0\right),(0, F, 0)\right\rangle_{\mathfrak{p}} \\
&-\frac{1}{2}\left\langle\left(0, L\left(K\left(X^{\prime}\right), Y^{\prime}\right)+L\left(Y^{\prime}, K\left(X^{\prime}\right)\right), 0\right),(0, F, 0)\right\rangle_{\mathfrak{p}} \\
&=-\frac{1}{2}\left\langle\left( 0,-L\left(X^{\prime}, K^{t}\left(Y^{\prime}\right)\right)+L\left(K\left(Y^{\prime}\right), X^{\prime}\right)+L\left(K\left(X^{\prime}\right), Y^{\prime}\right)\right.\right. \\
&\left.\left.\quad-L\left(Y^{\prime}, K^{t}\left(X^{\prime}\right)\right), 0\right),(0, F, 0)\right\rangle_{\mathfrak{p}} \\
&=-\frac{1}{2}\left\langle\left(0,\left[K, L\left(X^{\prime}, Y^{\prime}\right)\right]+\left[K, L\left(Y^{\prime}, X^{\prime}\right)\right], 0\right),(0, F, 0)\right\rangle_{\mathfrak{p}} \\
&=-\left\langle\left(0,\left[K, F^{\prime}\right], 0\right),(0, F, 0)\right\rangle_{\mathfrak{p}}
\end{aligned}
$$

by (5.5). Hence we have

$$
\begin{aligned}
\langle[(X, & \left.K, X),(Y, F,-Y)],\left(Y^{\prime}, F^{\prime},-Y^{\prime}\right)\right\rangle_{\mathfrak{p}} \\
= & \langle(K(Y)-F(X),[K, F]+(1 / 2)(L(X, Y)+L(Y, X)), \\
& \left.-K(Y)+F(X)),\left(Y^{\prime}, F^{\prime},-Y^{\prime}\right)\right\rangle_{\mathfrak{p}} \\
= & \left\langle K(Y)-F(X), Y^{\prime}\right\rangle+\left\langle F^{\prime}(X), Y\right\rangle+\left\langle(0,[K, F], 0),\left(0, F^{\prime}, 0\right)\right\rangle_{\mathfrak{p}} \\
= & -\left\langle K\left(Y^{\prime}\right), Y\right\rangle-\left(\left\langle F(X), Y^{\prime}\right\rangle-\left\langle F^{\prime}(X), Y\right\rangle\right) \\
& \quad-\left\langle\left(0,\left[K, F^{\prime}\right], 0\right),(0, F, 0)\right\rangle_{\mathfrak{p}} \\
= & -\left\langle\left[(X, K, X),\left(Y^{\prime}, F^{\prime},-Y^{\prime}\right)\right],(Y, F,-Y)\right\rangle_{\mathfrak{p}}
\end{aligned}
$$

for $(X, K, X) \in \mathfrak{f},(Y, F,-Y),\left(Y^{\prime}, F^{\prime},-Y^{\prime}\right) \in \mathfrak{p}$.

q.e.d.

We call this $\left(\mathfrak{g}=\sum \mathfrak{g}_{\mu}, \rho,\langle\rangle_{\mathrm{p}}\right.$ ) the OSGLA associated with an OJTS $(V,\{\},\langle\rangle)$, and denote the morphism by $\iota:(V,\{\},\langle\rangle) \rightarrow\left(\mathfrak{g}=\sum \mathfrak{g}_{\mu}\right.$, $\left.\rho,\langle\rangle_{p}\right)$.

Let $\left(\mathfrak{g}=\sum \mathfrak{g}_{\mu}, \rho\right)$ be an SGLA. Put $V=\mathfrak{g}_{-1}$ and define a $V$-valued trilinear form \{\} on $V$ by

$$
\{X, Y, Z\}=-2[[X, \rho(Y)], Z]
$$

for $X, Y, Z \in V$. Then $(V,\{\})$ is a JTS. In fact, we have

$$
\begin{aligned}
L(X, Y) Z & =-2[[X, \rho(Y)], Z]=-2[X,[\rho(Y), Z]] \\
& =-2[[Z, \rho(Y)], X]=L(Z, Y) X
\end{aligned}
$$

for $X, Y, Z \in V$. This implies (JT 1). Note that

$$
\begin{aligned}
{[\rho([X, \rho(Y)]), Z] } & =\rho([X, \rho(Y)], \rho(Z)])=-\rho([[\rho(Z), X], \rho(Y)]) \\
& =-[[Z, \rho(X)], Y]=-[[Y, \rho(X)], Z]
\end{aligned}
$$

for $X, Y, Z \in V$, and thus $\rho([X, \rho(Y)])=-[Y, \rho(X)]$ by (SGL 3). Then we have 


$$
\begin{aligned}
& {[L(X, Y), L(Z, W)]=4\left[\left.\operatorname{ad}([X, \rho(Y)])\right|_{V},\left.\operatorname{ad}([Z, \rho(W)])\right|_{V}\right]} \\
& \quad=\left.4 \operatorname{ad}([[X, \rho(Y)],[Z, \rho(W)]])\right|_{V} \\
& \quad=4\left\{\left.\operatorname{ad}([[[X, \rho(Y)], Z], \rho(W)])\right|_{V}+\left.\operatorname{ad}([Z,[[X, \rho(Y)], \rho(W)]])\right|_{V}\right\} \\
& \quad=L(L(X, Y) Z, W)-L(Z, L(Y, X) W)
\end{aligned}
$$

for $X, Y, Z, W \in V$. This implies (JT 2).

This $(V,\{\})$ is called the JTS associated with an SGLA $\left(\mathfrak{g}=\sum \mathfrak{g}_{\mu}, \rho\right)$.

Let $\left(\mathfrak{g}=\sum \mathfrak{g}_{\mu}, \rho\right)$ be an SGLA and $(V,\{\})$ the JTS associated with the SGLA. For a form \langle\rangle$_{\mathfrak{p}} \in \mathscr{S}(\mathrm{g}, \rho)$ define a symmetric bilinear form $\langle$ on $V$ by

$$
\langle X, Y\rangle=\langle X-\rho(X), Y-\rho(Y)\rangle_{p}
$$

for $X, Y \in V$.

LEMMA 5.3. Let $\langle>$ be a symmetric bilinear form on $V$ defined as above from \langle\rangle$_{\mathfrak{p}} \in \mathscr{S}(\mathfrak{g}, \rho)$. Then, $\langle>\in \mathscr{S}(V,\{\})$.

Proof. Note that

$$
\begin{aligned}
& \mathfrak{H}=\{X+\rho(X),[Y, \rho(Z)]-[Z, \rho(Y)] ; X, Y, Z \in V\}_{R}, \\
& \mathfrak{p}=\{X-\rho(X),[Y, \rho(Z)]+[Z, \rho(Y)] ; X, Y, Z \in V\}_{R}
\end{aligned}
$$

by (SGL 4). Define a trilinear form $\alpha$ on $V$ by

$$
\alpha(X, Y, Z)=\langle[X, \rho(Y)]+[Y, \rho(X)], Z-\rho(Z)\rangle_{\mathfrak{p}}
$$

for $X, Y, Z \in V$. Then we have

$$
\alpha(X, Y, Z)=\alpha(Y, X, Z) .
$$

Note that $[X, \rho(Y)]+[Y, \rho(X)]=-[X+\rho(X), Y-\rho(Y)]$ for $X, Y \in V$. Since $\left.\operatorname{ad}(T)\right|_{\mathfrak{p}}, T \in \mathfrak{F}$, are skew symmetric for \langle\rangle$_{\mathfrak{p}}$, we have

$$
\alpha(X, Y, Z)=-\alpha(X, Z, Y)
$$

and moreover

$$
\alpha(X, Y, Z)=\alpha(Z, Y, X)
$$

by (5.6), (5.7). Hence we have $\alpha=0$ by (5.6) (5.8). This implies that

$$
\left\langle\mathfrak{p} \cap \mathfrak{g}_{0}, \mathfrak{p} \cap\left(\mathfrak{g}_{-1} \oplus \mathfrak{g}_{1}\right)\right\rangle_{\mathfrak{p}}=\{0\} .
$$

Assume that $\langle X, Y\rangle=0$ for $Y \in V$. Then we have $\langle X-\rho(X), \mathfrak{p} \cap$ $\left.\left(\mathfrak{g}_{-1} \oplus \mathfrak{g}_{1}\right)\right\rangle_{\mathfrak{p}}=\{0\}$ by the definition of \langle\rangle , and thus $\langle X-\rho(X), \mathfrak{p}\rangle_{\mathfrak{p}}=\{0\}$ 
by (5.9). Since \langle\rangle$_{\mathfrak{p}}$ is non-degenerate, we have $X-\rho(X)=0$ and thus $X=0$. This implies that $\langle>$ is non-degenerate.

Finally we show that $L(X, Y)^{t}=L(Y, X)$ for $X, Y \in V$. Note that

$$
\begin{aligned}
& {[([X, \rho(Y)]-[Y, \rho(X)]), Z-\rho(Z)]} \\
& \quad=[([X, \rho(Y)]-[Y, \rho(X)]), Z]-\rho([([X, \rho(Y)]-[Y, \rho(X)]), Z])
\end{aligned}
$$

for $X, Y, Z \in V$. Then we have

$$
\begin{aligned}
& \langle\{L(X, Y)-L(Y, X)\} Z, W\rangle \\
& =(-2)\langle[([X, \rho(Y)]-[Y, \rho(X)]), Z-\rho(Z)], W-\rho(W)\rangle_{\mathfrak{p}} \\
& =2\langle Z-\rho(Z),[([X, \rho(Y)]-[Y, \rho(X)]), W-\rho(W)]\rangle_{\mathfrak{p}} \\
& =-\langle Z,\{L(X, Y)-L(Y, X)\} W\rangle
\end{aligned}
$$

for $X, Y, Z, W \in V$. This implies that $L(X, Y)-L(Y, X), X, Y \in V$, are skew symmetric for \langle\rangle . Note that

$$
\begin{aligned}
& {[Z+\rho(Z),[X, \rho(Y)]+[Y, \rho(X)]]} \\
& \quad=[Z,[X, \rho(Y)]+[Y, \rho(X)]]-\rho([Z,[X, \rho(Y)]+[Y, \rho(X)]])
\end{aligned}
$$

for $X, Y, Z \in V$. Then we have

$$
\begin{aligned}
& \langle\{L(X, Y)+L(Y, X)\} Z, W\rangle \\
& \quad=2\langle[Z+\rho(Z),[X, \rho(Y)]+[Y, \rho(X)]], W-\rho(W)\rangle_{\mathfrak{p}} \\
& \quad=(-2)\langle[X, \rho(Y)]+[Y, \rho(X)],[Z+\rho(Z), W-\rho(W)]\rangle_{\mathfrak{p}} \\
& \quad=2\langle[X, \rho(Y)]+[Y, \rho(X)],[Z, \rho(W)]+[W, \rho(Z)]\rangle_{\mathfrak{p}}
\end{aligned}
$$

for $X, Y, Z, W \in V$. This implies that $L(X, Y)+L(Y, X), X, Y \in V$, are symmetric for $\left\langle>\right.$. Hence we have $L(X, Y)^{t}=L(Y, X)$ for $X, Y \in V$.

q.e.d.

We call this $(V,\{\},\langle\rangle)$ the OJTS associated with an OSGLA $(\mathfrak{g}=$ $\left.\sum \mathfrak{g}_{\mu}, \rho,\langle\rangle_{\mathfrak{p}}\right)$ and denote the morphism by $\iota^{-1}:\left(\mathfrak{g}=\sum \mathfrak{g}_{\mu}, \rho,\langle\rangle_{p}\right) \rightarrow(V$, \{\}$,\langle\rangle)$.

TheOREM 5.4. (1) Two morphisms $\iota, \iota^{-1}$ are invertible to each other.

(2) Two OJTS's are equivalent to each other if and only if OSGLA's associated with them are equivalent to each other.

(3) Let $\left(V,\{\},\langle>)\right.$ be an OJTS and $\left(\mathfrak{g}=\sum \mathfrak{g}_{\mu}, \rho,\langle\rangle_{\mathrm{p}}\right)$ the OSGLA associated with $(V,\{\},\langle\rangle)$. Denote by $\beta$ the trace form of $(V,\{\})$, and by $B_{9}, B_{g_{0}}$ the Killing forms of $\mathfrak{g}, g_{0}$ respectively. Then,

$$
B_{8}((X, F, Y),(Z, G, W))=B_{\mathrm{g}_{0}}(F, G)+2 \operatorname{Tr} F G-(\beta(X, W)+\beta(Y, Z))
$$


for $(X, F, Y),(Z, G, W) \in \mathfrak{g}$. Moreover,

$$
B_{g}((X, F,-X),(Y, G,-Y))=2\left\{\sum_{i} \beta\left(G\left(U_{i}\right), V_{i}\right)+\beta(X, Y)\right\}
$$

for $(X, F,-X),(Y, G,-Y) \in \mathfrak{p}$, where $2 F=\sum_{i} L\left(U_{i}, V_{i}\right)+L\left(V_{i}, U_{i}\right)$.

(4) Let $(V,\{\},\langle\rangle)$ be an OJTS and $\left(\mathfrak{g}=\sum \mathfrak{g}_{\mu}, \rho,\langle\rangle_{\mathfrak{p}}\right)$ the OSGLA associated with $(V,\{\},\langle\rangle)$. Then the JTS $(V,\{\})$ is non-degenerate if and only if the $S L A(\mathfrak{g}, \rho)$ is semi-simple.

(5) Assume that a JTS $(V,\{\})$ is non-degenerate. Then the OSGLA associated with $(V,\{\quad\}, 2 \beta)$ is $\left(\mathfrak{g}=\sum \mathfrak{g}_{\mu}, \rho,\left.B_{\mathfrak{g}}\right|_{\mathfrak{p} \times \mathfrak{p}}\right)$.

Proof. (1) Let $(V,\{\},\langle\rangle)$ be an OJTS and $\left(\mathfrak{g}=\sum \mathfrak{g}_{\mu}, \rho,\langle\rangle_{\mathrm{p}}\right)$ the OSGLA associated with $(V,\{\},\langle\rangle)$. Moreover let $\left(V^{\prime},\{\}^{\prime},\langle\rangle^{\prime}\right)$ be the OJTS associated with $\left(\mathfrak{g}=\sum \mathfrak{g}_{\mu}, \rho,\langle\rangle_{\mathfrak{p}}\right)$. Then we show that two OJTS's $\left(V,\{\},\langle>),\left(V^{\prime},\{\}^{\prime},\langle\rangle^{\prime}\right)\right.$ are equivalent to each other. Identify $V$ with $V^{\prime}$ by $V \ni X \rightarrow(X, 0,0) \in g_{-1}=V^{\prime}$. Then we have

$$
\{X, Y, Z\}^{\prime}=-2[[X, \rho(Y)], Z]=L(X, Y) Z=\{X, Y, Z\}
$$

for $X, Y, Z \in V$, and

$$
\langle X, Y\rangle^{\prime}=\langle X-\rho(X), Y-\rho(Y)\rangle_{\mathfrak{p}}=\langle X, Y\rangle
$$

for $X, Y \in V$. Hence OJTS's $(V,\{\},\langle\rangle),\left(V^{\prime},\{\}^{\prime},\langle\rangle^{\prime}\right)$ are equivalent to each other.

Conversely, let $\left(\mathfrak{g}=\sum \mathfrak{g}_{\mu}, \rho,\langle\rangle_{\mathfrak{p}}\right)$ be an OSGLA and $(V,\{\},\langle\rangle)$ the OJTS associated with $\left(\mathfrak{g}=\sum \mathfrak{g}_{\mu}, \rho,\langle\rangle_{\mathfrak{p}}\right)$. Moreover let $\left(\mathfrak{g}^{\prime}=\sum \mathfrak{g}_{\mu}^{\prime}, \rho^{\prime}\right.$, \langle\rangle$\left._{p^{\prime}}\right)$ be the OSGLA associated with $(V,\{\},\langle\rangle)$. Then we show that two OSGLA's $\left(g=\sum g_{\mu}, \rho,\langle\rangle_{p}\right),\left(g^{\prime}=\sum g_{\mu}^{\prime}, \rho^{\prime},\langle\rangle_{\mathfrak{p}^{\prime}}\right)$ are equivalent to each other. Note that $\mathfrak{g}_{0}^{\prime}=\left\{\left(0\right.\right.$, ad $\left.\left.(T) \mid g_{-1}, 0\right) ; T \in \mathfrak{g}_{0}\right\}$ by (SGL 4$)$ for $(\mathfrak{g}=$ $\left.\sum \mathfrak{g}_{\mu}, \rho\right)$. Define a linear mapping $\tau$ of $g$ onto $\mathrm{g}^{\prime}$ by $\tau(X+T+\rho(Y))=(X$, ad $\left.(T) \mid \mathfrak{g}_{-1}, Y\right)$ for $X, Y \in \mathfrak{g}_{-1}, T \in \mathfrak{g}_{0}$. Then $\tau$ is injective by (SGL 3) for $\left(\mathfrak{g}=\sum \mathfrak{g}_{\mu}, \rho\right) . \quad$ Since $L(X, Y)=-2$ ad $\left.([X, \rho(Y)])\right|_{s_{-1}}$ and $L(X, Y)^{t}=L(Y, X)$ for $X, Y \in V=g_{-1}$, we have

$$
\left(\left.\operatorname{ad}(T)\right|_{g_{-1}}\right)^{t}=-\left.\operatorname{ad}(\rho(T))\right|_{g_{-1}}
$$

for $T \in \mathfrak{f}$ by (SGL 4) for $\left(\mathfrak{g}=\sum \mathfrak{g}_{\mu}, \rho\right)$. Then we have

$$
\begin{aligned}
\tau([X+ & T+\rho(Y), Z+S+\rho(W)])=\tau\{([X, S]+[T, Z]) \\
& +([T, S]+[X, \rho(W)]+[\rho(Y), Z])+([\rho(Y), S]+[T, \rho(W)])\} \\
= & \left([X, S]+[T, Z],\left.\operatorname{ad}([T, S])\right|_{g_{-1}}+\left.\operatorname{ad}([X, \rho(W)])\right|_{g_{-1}}\right. \\
& \left.+\left.\operatorname{ad}([\rho(Y), Z])\right|_{g_{-1}},[Y, \rho(S)]+[\rho(T), W]\right)
\end{aligned}
$$




$$
\begin{aligned}
= & \left(\left.\operatorname{ad}(T)\right|_{g_{-1}}(Z)-\left.\operatorname{ad}(S)\right|_{g_{-1}}(X),\left[\left.\operatorname{ad}(T)\right|_{g_{-1}},\left.\operatorname{ad}(S)\right|_{g_{-1}}\right]\right. \\
& \left.-(1 / 2)(L(X, W)-L(Z, Y)),\left(\left.\operatorname{ad}(S)\right|_{g_{-1}}\right)^{t} Y-\left(\left.\operatorname{ad}(T)\right|_{g_{-1}}\right)^{t} W\right) \\
= & {\left[\left(X,\left.\operatorname{ad}(T)\right|_{g_{-1}}, Y\right),\left(Z,\left.\operatorname{ad}(S)\right|_{g_{-1}}, W\right)\right] } \\
= & {[\tau(X+T+\rho(Y)), \tau(Z+S+\rho(W))] }
\end{aligned}
$$

by (5.1), (5.10), and thus $\tau$ is a Lie algebra homomorphism. Since $\tau\left(\mathfrak{g}_{\mu}\right)$ $=\mathfrak{g}_{\mu}^{\prime}$ for $\mu=0, \pm 1, \tau$ is an SGLA-isomorphism. Moreover we have

$$
\begin{aligned}
& (\tau \circ \rho)(X+T+\rho(Y))=\tau(Y+\rho(T)+\rho(X))=\left(Y,\left.\operatorname{ad}(\rho(T))\right|_{g_{-1}}, X\right) \\
& \quad=\left(Y,-\left(\left.\operatorname{ad}(T)\right|_{g_{-1}}\right)^{t}, X\right)=\rho^{\prime}\left(X,\left.\operatorname{ad}(T)\right|_{g_{-1}}, Y\right)=\rho^{\prime} \circ \tau(X+T+\rho(Y))
\end{aligned}
$$

for $X, Y \in \mathfrak{g}_{-1}, T \in \mathfrak{f}$ by (5.2), (5.10), and thus $\tau \circ \rho=\rho^{\prime} \circ \tau$. Let $T, S \in \mathfrak{p} \cap \mathfrak{g}_{0}$. and put $T=\sum_{i}\left[Y_{i}, \rho\left(Z_{i}\right)\right]+\left[Z_{i}, \rho\left(Y_{i}\right)\right]$. Since ad $\left.(U)\right|_{\mathfrak{p}}, U \in \mathfrak{f}$, are skew symmetric for \langle\rangle$_{p}$, we have

$$
\begin{aligned}
\langle T, S\rangle_{\mathfrak{p}} & =\sum_{i}\left\langle\left[Y_{i}, \rho\left(Z_{i}\right)\right]+\left[Z_{i}, \rho\left(Y_{i}\right)\right], S\right\rangle_{\mathfrak{p}} \\
& =-\sum_{i}\left\langle\left[Y_{i}+\rho\left(Y_{i}\right), Z_{i}-\rho\left(Z_{i}\right)\right], S\right\rangle_{\mathfrak{p}} \\
& =\sum_{i}\left\langle Z_{i}-\rho\left(Z_{i}\right),\left[Y_{i}+\rho\left(Y_{i}\right), S\right]\right\rangle_{\mathfrak{p}} \\
& =-\sum_{i}\left\langle Z_{i}-\rho\left(Z_{i}\right),\left[S, Y_{i}\right]-\rho\left(\left[S, Y_{i}\right]\right)\right\rangle_{\mathfrak{p}} \\
& =-\sum_{i}\left\langle Z_{i},\left.\operatorname{ad}(S)\right|_{\mathfrak{g}_{-1}}\left(Y_{i}\right)\right\rangle .
\end{aligned}
$$

Note that $\left.\operatorname{ad}(T)\right|_{s_{-1}}=(1 / 2) \sum_{i} L\left(-Y_{i}, Z_{i}\right)+L\left(Z_{i},-Y_{i}\right)$. Then we have

$$
\begin{aligned}
\langle\tau(X & +T-\rho(X)), \tau(Y+S-\rho(Y))\rangle_{p^{\prime}} \\
& =\left\langle\left(X,\left.\operatorname{ad}(T)\right|_{g_{-1}},-X\right),\left(Y,\left.\operatorname{ad}(S)\right|_{q_{-1}},-Y\right)\right\rangle_{p^{\prime}} \\
& =\langle X, Y\rangle-\sum\left\langle\left.\operatorname{ad}(S)\right|_{g_{-1}}(Y), Z_{i}\right\rangle=\langle X, Y\rangle+\langle T, S\rangle \\
& =\langle X+T-\rho(X), Y+S-\rho(Y)\rangle_{\mathfrak{p}}
\end{aligned}
$$

for $X, Y \in \mathfrak{g}_{-1}, \quad T, \quad S \in \mathfrak{p} \cap \mathfrak{g}_{0}$. Hence $\left(\mathfrak{g}=\sum \mathfrak{g}_{\mu}, \rho,\langle\rangle_{\mathfrak{p}}\right), \quad\left(\mathfrak{g}^{\prime}=\sum \mathfrak{g}_{\mu}^{\prime}, \rho^{\prime}\right.$, \langle\rangle$_{p^{\prime}}$ ) are equivalent to each other.

(2) Let $(V,\{\},\langle\rangle),\left(V^{\prime},\{\}^{\prime},\langle\rangle^{\prime}\right)$ be OJTS's and $\left(\mathfrak{g}=\sum \mathfrak{g}_{\mu}, \rho\right.$, \langle\rangle$\left._{\mathfrak{p}}\right),\left(\mathfrak{g}^{\prime}=\sum \mathfrak{g}_{\mu}^{\prime}, \rho^{\prime},\langle\rangle_{\mathfrak{p}^{\prime}}\right)$ the OSGLA's associated with $(V,\{\},\langle\rangle)$, $\left(V^{\prime},\{\}^{\prime},\langle\rangle^{\prime}\right)$ respectively. Assume that $(V,\{\},\langle\rangle),\left(V^{\prime},\{\}^{\prime},\langle\rangle^{\prime}\right)$ are equivalent to each other, i.e., there exists a linear isomorphism $g$ of $V$ onto $V^{\prime}$ such that $\{g(X), g(Y), g(Z)\}^{\prime}=g\{X, Y, Z\},\langle g(X), g(Y)\rangle^{\prime}=\langle X$, $Y>$ for $X, Y, Z \in V$. Note that

$$
L^{\prime}(g(X), g(Y))=g \circ L(X, Y) \circ g^{-1}, \quad\left(g \circ F \circ g^{-1}\right)^{t}=g \circ F^{t} \circ g^{-1}
$$

for $X, Y \in V, F \in L$. Define a linear isomorphism $\tau_{g}$ of $\mathfrak{g}$ onto $\mathfrak{g}^{\prime}$ by $\tau_{g}(X$, $F, Y)=\left(g(X), g \circ F \circ g^{-1}, g(Y)\right)$ for $(X, F, Y) \in \mathfrak{g}$. Then we have 


$$
\begin{aligned}
\tau_{g}([(X, & F, Y),(Z, G, W)])=(g(F(Z)-G(X)), g \circ([F, G]-(1 / 2) L(X, W) \\
& \left.+(1 / 2) L(Z, Y)) \circ g^{-1}, g\left(G^{t}(Y)-F^{t}(W)\right)\right) \\
= & \left(\left(g \circ F \circ g^{-1}\right)(g(Z))-\left(g \circ G \circ g^{-1}\right)(g(X)),\left[g \circ F \circ g^{-1}, g \circ G \circ g^{-1}\right]\right. \\
& -(1 / 2) L^{\prime}(g(X), g(W))+(1 / 2) L^{\prime}(g(Z), g(Y)),\left(g \circ G \circ g^{-1}\right)^{t}(g(Y)) \\
& \left.-\left(g \circ F \circ g^{-1}\right)^{t}(g(W))\right) \\
= & {\left[\left(g(X), g \circ F \circ g^{-1}, g(Y)\right),\left(g(Z), g \circ G \circ g^{-1}, g(W)\right)\right] } \\
= & {\left[\tau_{g}(X, F, Y), \tau_{g}(Z, G, W)\right] }
\end{aligned}
$$

for $(X, F, Y),(Z, G, W) \in \mathfrak{g}$, and thus $\tau_{g}$ is a Lie algebra isomorphism. Since $\tau_{g}\left(\mathfrak{g}_{\mu}\right)=\mathfrak{g}_{\mu}^{\prime}$ for $\mu=0, \pm 1, \tau_{g}$ is an SGLA-isomorphism. Moreover we have

$$
\begin{aligned}
\left(\tau_{g} \circ \rho\right)(X, F, Y) & =\tau_{g}\left(Y,-F^{t}, X\right)=\left(g(Y),-g \circ F^{t} \circ g^{-1}, g(X)\right) \\
& =\left(g(Y),-\left(g \circ F \circ g^{-1}\right)^{t}, g(X)\right)=\rho^{\prime} \circ \tau_{g}(X, F, Y)
\end{aligned}
$$

for $(X, F, Y) \in \mathfrak{g}$. Then it is straightforwardly proved by (5.11) that $\tau_{g}$ is an OSGLA-isomorphism of $\mathfrak{g}$ onto $\mathfrak{g}^{\prime}$. Note that $2 g \circ G \circ g^{-1}=\sum_{i} L\left(g\left(U_{i}\right)\right.$, $\left.g\left(V_{i}\right)\right)+L\left(g\left(V_{i}\right), g(U)\right)$ for $2 G=\sum_{i} L\left(U_{i}, V_{i}\right)+L\left(V_{i}, U_{i}\right) \in g_{0} \cap \mathfrak{p}$ by (5.11). Then we have

$$
\begin{aligned}
\left\langle\tau_{g}(X,\right. & \left.F,-X), \tau_{g}(Y, G,-Y)\right\rangle_{\mathfrak{p}^{\prime}} \\
\quad & \left\langle\left(g(X), g \circ F \circ g^{-1},-g(X)\right),\left(g(Y), g \circ G \circ g^{-1},-g(Y)\right)\right\rangle_{\mathfrak{p}^{\prime}} \\
& =\langle g(X), g(Y)\rangle+\sum_{i}\left\langle g \circ F \circ g^{-1}\left(g\left(U_{i}\right)\right), g\left(V_{i}\right)\right\rangle \\
& =\langle X, Y\rangle+\sum_{i}\left\langle F\left(U_{i}\right), V_{i}\right\rangle=\langle(X, F,-X),(Y, G,-Y)\rangle_{\mathfrak{p}}
\end{aligned}
$$

for $(X, F,-X),(Y, G,-Y) \in \mathfrak{p}$. Hence OSGLA's $\left(\mathfrak{g}=\sum \mathfrak{g}_{\mu}, \rho,\langle\rangle_{\mathfrak{p}}\right),\left(\mathfrak{g}^{\prime}=\right.$ $\left.\sum g_{\mu}^{\prime}, \rho^{\prime},\langle\rangle_{p^{\prime}}\right)$ are equivalent to each other.

Conversely, assume that OSGLA's $\left(\mathfrak{g}=\sum \mathfrak{g}_{\mu}, \rho,\langle\rangle_{p}\right),\left(\mathfrak{g}^{\prime}=\sum \mathfrak{g}_{\mu}^{\prime}, \rho^{\prime}\right.$, \langle\rangle$_{D^{\prime}}$ ) are equivalent to each other, i.e., there exists an SGLA-isomorphism $\tau$ of $\left(\mathfrak{g}=\sum \mathrm{g}_{\mu}, \rho^{\prime}\right)$ onto $\left(\mathrm{g}^{\prime}=\sum \mathrm{g}_{\mu}^{\prime}, \rho^{\prime}\right)$ such that $\langle\tau(A), \tau(B)\rangle_{\mathrm{p}^{\prime}}=\langle A, B\rangle_{\mathrm{p}}$ for $A, B \in \mathfrak{p}$. Define a linear isomorphism $g_{\imath}$ of $V=\mathfrak{g}_{-1}$ onto $V^{\prime}=\mathfrak{g}_{-1}^{\prime}$ by $g_{r}(X)=\tau(X)$ for $X \in V=\mathfrak{g}_{-1}$. Then we have

$$
\begin{aligned}
& \left\{g_{\tau}(X), g_{\tau}(Y), g_{\tau}(Z)\right\}^{\prime}=-2\left[\left[g_{\tau}(X), \rho^{\prime}\left(g_{\tau}(Y)\right)\right], g_{\tau}(Z)\right] \\
& \quad=-2[[\tau(X), \tau(\rho(Y))], \tau(Z)]=\tau(-2[[X, \rho(Y)], Z])=g_{\tau}\{X, Y, Z\}
\end{aligned}
$$

and

$$
\begin{aligned}
& \left\langle g_{\tau}(X), g_{\tau}(Y)\right\rangle^{\prime}=\left\langle\tau(X)-\rho^{\prime}(\tau(X)), \tau(Y)-\rho^{\prime}(\tau(Y))\right\rangle_{\mathfrak{p}^{\prime}} \\
& \quad=\langle\tau(X-\rho(X)), \tau(Y-\rho(Y))\rangle_{p^{\prime}}=\langle X-\rho(X), Y-\rho(Y)\rangle_{\mathfrak{p}}=\langle X, Y\rangle
\end{aligned}
$$


for $X, Y, Z \in V$. Hence OJTS's $(V,\{\},\langle\rangle),\left(V^{\prime},\{\}^{\prime},\langle\rangle^{\prime}\right)$ are equivalent to each other.

(3) Note that $\operatorname{Tr} L(X, Y)=\operatorname{Tr} L(Y, X)^{t}=\operatorname{Tr} L(Y, X)$ for $X, \quad Y \in V$. Then the first identity is proved in the same way as in Koecher [8], II, $\S 4$.

Now we show the second identity. Since $(0, F, 0)=\sum_{i}\left[\left(U_{i}, 0, U_{i}\right)\right.$, $\left.\left(V_{i}, 0,-V_{i}\right)\right]$, we have

$$
\begin{array}{r}
B_{\mathrm{q}}((0, F, 0),(0, G, 0))=-\sum_{i} B_{g}\left(\left(V_{i}, 0,-V_{i}\right),\left[\left(U_{i}, 0, U_{i}\right),(0, G, 0)\right]\right) \\
=\sum_{i} B_{9}\left(\left(V_{i}, 0,-V_{i}\right),\left(G\left(U_{i}\right), 0,-G\left(U_{i}\right)\right)\right)=2 \sum_{i} \beta\left(G\left(U_{i}\right), V_{i}\right)
\end{array}
$$

and thus

$$
\begin{aligned}
B_{\mathrm{g}}((X, F,-X),(Y, G,-Y)) & =B_{9}((0, F, 0),(0, G, 0))+2 \beta(X, Y) \\
& =2\left\{\sum_{i} \beta\left(G\left(U_{i}\right), V_{i}\right)+\beta(X, Y)\right\}
\end{aligned}
$$

for $(X, F,-X),(Y, G,-Y) \in \mathfrak{p}$.

(4) Assume that $(V,\{\})$ is non-degenerate and that $B_{g}((X, F, Y)$, $(Z, G, W))=0$ for $(X, F, Y) \in \mathfrak{g}$. Putting $F=0, X=0$ (resp. $F=0, Y=0)$, we have $\beta(Y, Z)=0$ for $Y \in V$ (resp. $\beta(X, W)=0$ for $X \in V)$ by (3). These imply that $Z=W=0$. Note that $(0, L(A, B), 0)=-2[(A, 0,0),(0,0, B)]$ for ${ }^{*} A, B \in V$. Then we have

$$
\begin{aligned}
0 & =B_{9}((0, G, 0),(0, L(A, B), 0))=-2 B_{\mathrm{g}}((0, G, 0),[(A, 0,0),(0,0, B)]) \\
& =2 B_{\mathrm{g}}([(A, 0,0),(0, G, 0)],(0,0, B))=-2 B_{9}((G(A), 0,0),(0,0, B)) \\
& =2 \beta(G(A), B)
\end{aligned}
$$

by (3). This implies that $G=0$. Hence $g$ is semi-simple.

Conversely, assume that $(V,\{\})$ is degenerate, i.e., there exists a nonzero vector $Z \in V$ such that $\beta(Z, Y)=0$ for $Y \in V$. Then we have $B_{g}((Z, 0,0),(X, F, Y))=-\beta(Z, Y)=0$ for $(X, F, Y) \in g$ by $(3)$. This implies that $g$ is not semi-simple.

The claim (5) is obvious by (3).

q.e.d.

A finite dimensional algebra $A$ over $\boldsymbol{R}$ is called a Jordan algebra (abbreviated as JA) if the following two conditions are satisfied:

(J 1) $X \cdot Y=Y \cdot X$

(J 2) $X^{2} \cdot(X \cdot Y)=X \cdot\left(X^{2} \cdot Y\right)$

for $X, Y \in A$. Define linear endomorphisms $T_{X}, X \in A$, of $A$ by $T_{X}(Y)=$ 
$X \cdot Y$ for $Y \in A$. Put $V_{A}=A$ and define a $V_{A}$-valued trilinear form \{\}$_{A}$ on $V_{A}$ by

$$
\{X, Y, Z\}_{A}=(X \cdot Y) \cdot Z+X \cdot(Y \cdot Z)-Y \cdot(X \cdot Z)
$$

for $X, Y, Z \in V_{A}$. Then the object $\left(V_{A},\{\}_{A}\right)$ is a JTS (cf. Satake [16]). This $\left(V_{A},\{\}_{A}\right)$ is called the JTS coming from a JA $A$. If $A$ has the unity $E$, we can recover the Jordan product - by $X \cdot Y=\{X, Y, E\}_{A}=\{X, E, Y\}_{A}$ $=\{E, X, Y\}_{A}$. Denote by $\mathscr{S}(A)$ the set of non-degenerate symmetric bilinear forms $\left\langle>\right.$ on $A$ such that $T_{X}, X \in A$, are symmetric for $\langle>$. An object $(A,\langle\rangle)$ is called an orthogonal Jordan algebra (abbreviated as OJA) for a JA $A$ and a form $\left\langle>\in \mathscr{S}(A)\right.$. Note that $\mathscr{S}(A) \subset \mathscr{S}\left(V_{A},\{\}_{A}\right)$. In fact, let $\left\langle>\in \mathscr{S}(A)\right.$. Since $L_{A}(X, Y)=T_{X \cdot Y}+\left[T_{X}, T_{Y}\right]$ by (5.12), we have

$$
\begin{aligned}
\left\langle L_{A}(X, Y) Z, W\right\rangle & =\left\langle T_{X \cdot Y}(Z), W\right\rangle+\left\langle\left[T_{X}, T_{Y}\right](Z), W\right\rangle \\
& =\left\langle Z, T_{Y \cdot X}(W)\right\rangle+\left\langle Z,\left[T_{Y}, T_{X}\right](W)\right\rangle=\left\langle Z, L_{A}(Y, X) W\right\rangle
\end{aligned}
$$

for $X, Y, Z, W \in A=V_{A}$, and thus $L_{A}(X, Y)^{t}=L_{A}(Y, X)$. This implies that $\left\langle>\in \mathscr{S}\left(V_{A},\{\}_{A}\right)\right.$. We call this $\left(V_{A},\{\}_{A},\langle\rangle\right)$ the OJTS coming from an OJA $(A,\langle\rangle)$. Moreover assume that $A$ has the unity $E$. Then $\mathscr{S}(A)$ $=\mathscr{S}\left(V_{A},\{\}_{A}\right)$. In fact, let $\left\langle>\in \mathscr{S}\left(V_{A},\{\}_{A}\right)\right.$. Since $T_{E}=\mathrm{id}_{A}$, we have

$$
\begin{aligned}
\left\langle T_{X}(Z), W\right\rangle & =\left\langle\left(T_{X \cdot E}+\left[T_{X}, T_{E}\right]\right)(Z), W\right\rangle=\left\langle L_{A}(X, E) Z, W\right\rangle \\
& =\left\langle Z, L_{A}(E, X) W\right\rangle=\left\langle Z, T_{X}(W)\right\rangle
\end{aligned}
$$

for $X, Z, W \in A=V_{A}$.

Now it is said that two OJA's $(A,\langle\rangle),\left(A^{\prime},\langle\rangle^{\prime}\right)$ are equivalent to each other if there exists an algebra isomorphism $g$ of $A$ onto $A^{\prime}$ such that $\langle g(X), g(Y)\rangle^{\prime}=\langle X, Y\rangle$ for $X, Y \in A$. If two OJA's are equivalent to each other, the OJTS's coming from them are equivalent to each other. But the converse is not necessarily true.

Let $\left(V_{A},\{\}_{A},\langle\rangle\right)$ be the OJTS coming from an OJA $(A,\langle\rangle)$ with unity $E$, and $\left(\mathfrak{g}_{A}=\sum\left(\mathfrak{g}_{A}\right)_{\mu}, \rho_{A},\langle\rangle_{\mathfrak{p}_{A}}\right)$ the OSGLA associated with $\left(V_{A},\{\}_{A}\right.$, \langle\rangle ). (For simplicity, we call this $\left(\mathfrak{g}_{A}=\sum\left(\mathfrak{g}_{A}\right)_{\mu}, \rho_{A},\langle\rangle_{\mathfrak{p}_{A}}\right)$ the OSGLA coming from $(A,\langle\rangle)$ ) $)$ Put $J=(E, 0, E) \in \mathfrak{l}_{A}$ and $J_{p_{A}}=\operatorname{ad}(J) \mid \mathfrak{p}_{A}$.

THEOREM 5.5. (1) Let $(A,\langle\rangle)$ be an OJA with unity $E$ and $\left(\mathfrak{g}_{A}=\right.$ $\left.\sum\left(\mathfrak{g}_{A}\right)_{\mu}, \rho_{A},\langle\rangle_{p_{A}}\right)$ the OSGLA coming from $(A,\langle\rangle)$. Then $\left(\mathfrak{g}_{A}=\sum\left(\mathfrak{g}_{A}\right)_{\mu}\right.$, $\rho_{A}, J_{p_{A}},\langle\rangle_{p_{A}}$ ) is always an effective HSGLA such that $L_{A} \ni \mathrm{id}_{A^{\prime}}$.

(2) Let $(A,\langle\rangle),\left(A^{\prime},\langle\rangle^{\prime}\right)$ be OJA's with unities $E, E^{\prime}$, and $\left(\mathfrak{g}_{A}=\right.$ 
$\left.\left.\left.\sum\left(\mathfrak{g}_{A}\right)_{\mu}, \rho_{A}, J_{p_{A}}<\right\rangle_{p_{A}}\right),\left(\mathfrak{g}_{A^{\prime}}=\sum\left(\mathfrak{g}_{A^{\prime}}\right)_{\mu}, \rho_{A^{\prime}}, J_{p_{A^{\prime}},}<\right\rangle_{p_{A_{A}}}\right)$ the HSGLA's coming from $(A,\langle\rangle),\left(A^{\prime},\langle\rangle^{\prime}\right)$ respectively. Then $(A,\langle\rangle),\left(A^{\prime},\langle\rangle^{\prime}\right)$ are equivalent to each other if and only if $\left(\mathfrak{g}_{A}=\sum\left(\mathfrak{g}_{A}\right)_{\mu}, \rho_{A}, J_{p_{A}},\langle\rangle_{p_{A}}\right),\left(\mathfrak{g}_{A^{\prime}}=\right.$ $\left.\sum\left(g_{A^{\prime}}\right)_{\mu}, \rho_{A^{\prime}}, J_{p_{A},},\langle\rangle_{p_{A} A^{\prime}}\right)$ are equivalent to each other.

Proof. (1) Note that $T_{X \cdot Y}$ are symmetric and $\left[T_{X}, T_{Y}\right]$ are skew symmetric in identities: $L_{A}(X, Y)=T_{X \cdot Y}+\left[T_{X}, T_{Y}\right], X, Y \in A$. Then we have

$$
\left\{\begin{array}{l}
\left(\mathfrak{g}_{A}\right)_{0} \cap \mathfrak{p}_{A}=\left\{\left(0, T_{X \cdot Y}, 0\right) ; X, Y \in A\right\}=\left\{\left(0, T_{X}, 0\right) ; X \in A\right\} \\
\left.\left(\mathfrak{g}_{A}\right)_{0} \cap \mathfrak{\mathfrak { f }}_{A}=\left(0,\left[T_{X}, T_{Y}\right], 0\right) ; X, Y \in A\right\}
\end{array}\right.
$$

since $A$ has the unity $E$. Now we have

$$
\begin{aligned}
& J_{p_{A}}\left(\left(X, T_{Y},-X\right)\right)=\left[(E, 0, E),\left(X, T_{Y},-X\right)\right] \\
& \quad=\left(-T_{Y}(E),(1 / 2)\{L(E, X)+L(X, E)\}, T_{Y}(E)\right)=\left(-Y, T_{X}, Y\right)
\end{aligned}
$$

for $\left(X, T_{Y},-X\right) \in \mathfrak{p}_{A}$ and thus $\left(J_{p_{A}}\right)^{2}=-\mathrm{id}_{p_{A}}$. Since

$$
\begin{aligned}
& {\left[(E, 0, E),\left(X,\left[T_{Y}, T_{Z}\right], X\right)\right]} \\
& \quad=\left(-\left[T_{Y}, T_{Z}\right](E),-(1 / 2)\{L(E, X)-L(X, E)\},\left[T_{Y}, T_{Z}\right](E)\right) \\
& \quad=(0,0,0)=\mathbf{0},
\end{aligned}
$$

$\boldsymbol{J}=(E, 0, E)$ is contained in the center of $\mathfrak{f}_{A}$ and thus ad $(\boldsymbol{U}) \mid \mathfrak{p}_{A} \circ J_{p_{A}}=$ $J_{\mathfrak{p}_{A}} \circ$ ad $(U) \mid \mathfrak{p}_{A}$ for $U \in \mathfrak{f}_{A}$. Hence $\left(\mathfrak{g}_{A}=\sum\left(\mathfrak{g}_{A}\right)_{\mu}, \rho_{A}, J_{p_{A}},\langle\rangle_{p_{A}}\right)$ is an HSGLA.

Since $L_{A}(E, E)=\mathrm{id}_{A}$, we have $\mathrm{id}_{A} \in L_{A}$. We show that $\left(\mathfrak{g}_{A}, \rho_{A}\right)$ is effective. Let $(X, F, X) \in \mathfrak{f}_{A}$ and assume that $[(X, F, X),(Y, G,-Y)]=0$ for $(Y$, $G,-Y) \in \mathfrak{p}_{A}$. Putting $Y=0, G=\mathrm{id}_{A}$, we have $(X, 0,-X)=0$ and thus $X=0$. Moreover, putting $G=0$, we have $F(Y)=0$ for $Y \in A=V_{A}$ and thus $F=0$. Hence $\left(\mathfrak{g}_{A}, \rho_{A}\right)$ is effective.

(2) Assume that $(A,\langle\rangle),\left(A^{\prime},\langle\rangle^{\prime}\right)$ are equivalent to each other, i.e., there exists an algebra isomorphism $\alpha$ of $A$ onto $A^{\prime}$ such that $\langle\alpha(X)$, $\alpha(Y)\rangle^{\prime}=\langle X, Y\rangle$ for $X, Y \in A$. Note that $\alpha(E)=E^{\prime}$. Define a linear isomorphism $g_{\alpha}$ of $V_{A}$ onto $V_{A^{\prime}}$ by $g_{\alpha}(X)=\alpha(X)$ for $X \in V_{A}=A$. Then $\left(V_{A}\right.$, \{\}$\left._{A},\langle\rangle\right)$ is equivalent to $\left(V_{A^{\prime}},\{\}_{A^{\prime}},\langle\rangle^{\prime}\right)$ by $g_{\alpha}$. Hence, by Theorem 5.4, (2), $\left(\mathfrak{g}_{A}=\sum\left(\mathfrak{g}_{A}\right)_{\mu}, \rho_{A},\langle\rangle_{\mathrm{p}_{A}}\right)$ is equivalent to $\left(\mathfrak{g}_{A^{\prime}}=\sum\left(\mathfrak{g}_{A^{\prime}}\right)_{\mu}, \rho_{A^{\prime}},\langle\rangle_{\mathrm{P}_{A^{\prime}}}\right)$ by $\tau_{g_{\alpha}}$. Since $\tau_{g_{\alpha}}(E, 0, E)=\left(E^{\prime}, 0, E^{\prime}\right)$, we have

$$
\tau_{g_{\alpha}} \circ J_{p_{A}}=\left.\tau_{g_{\alpha}} \circ \operatorname{ad}((E, 0, E))\right|_{p_{A}}=\left.\operatorname{ad}\left(\left(E^{\prime}, 0, E^{\prime}\right)\right)\right|_{p_{A^{\prime}}} \circ \tau_{g_{\alpha}}=J_{p_{A^{\prime}}} \circ \tau_{g_{\alpha}} .
$$

Hence HSGLA's $\left(\mathfrak{g}_{A}=\sum\left(\mathfrak{g}_{A}\right)_{\mu}, \rho_{A}, J_{p_{A}},\langle\rangle_{p_{A}}\right),\left(\mathfrak{g}_{A^{\prime}}=\sum\left(\mathfrak{g}_{A^{\prime}}\right)_{\mu}, \rho_{A^{\prime}}, J_{p_{A^{\prime}}},\langle\rangle_{p_{A^{\prime}}}\right)$ are equivalent to each other. 
Conversely, assume that $\left(\mathfrak{g}_{A}=\sum\left(\mathfrak{g}_{A}\right)_{\mu}, \rho_{A}, J_{p_{A}},\langle\rangle_{p_{A}}\right),\left(\mathfrak{g}_{A^{\prime}}=\sum\left(\mathfrak{g}_{A^{\prime}}\right)_{\mu}\right.$, $\rho_{A^{\prime}}, J_{p_{A^{\prime}}},\langle\rangle_{p^{\prime}}$ ) are equivalent to each other, i.e., there exists an SGLAisomorphism $\tau$ of $\left(g_{A}, \rho_{A}\right)$ onto $\left(g_{A^{\prime}}, \rho_{A^{\prime}}\right)$ such that $\tau \circ J_{p_{A}}=J_{p_{A^{\prime}}} \circ \tau,\langle\tau(A), \tau(B)\rangle_{p_{A^{\prime}}}$ $=\langle A, B\rangle_{\mathfrak{p}_{A}}$ for $\boldsymbol{A}, \boldsymbol{B} \in \mathfrak{p}_{A}$. Since $\tau$ is a Lie algebra isomorphism, we have $\left.\operatorname{ad}\left(\left(E^{\prime}, 0, E^{\prime}\right)\right) \circ \tau\right|_{p_{A}}=\left.\tau \circ \operatorname{ad}((E, 0, E))\right|_{p_{A}}=\left.\operatorname{ad}(\tau(E, 0, E)) \circ \tau\right|_{p_{A}}$.

Note that $\tau\left(\mathfrak{f}_{A}\right)=\mathfrak{f}_{A^{\prime}}, \tau\left(\mathfrak{p}_{A}\right)=\mathfrak{p}_{A^{\prime}}$. Since $\left(\mathfrak{g}_{A^{\prime}}, \rho_{A^{\prime}}\right)$ is effective, we have

$$
\tau((E, 0, E))=\left(E^{\prime}, 0, E^{\prime}\right) .
$$

Now, by Theorem 5.4, (2), $\left(V_{A},\{\}_{A},\langle\rangle\right)$ is equivalent to $\left(V_{A^{\prime}},\{\}_{A^{\prime}}\right.$, \langle\rangle$^{\prime}$ ) by $g_{\tau}$. Then $g_{\tau}(E)=E^{\prime}$ by (5.14). Define a linear isomorphism $\alpha$ of $A$ onto $A^{\prime}$ by $\alpha(X)=g_{\tau}(X)$ for $X \in A=V_{A}$. Then we have

$$
\begin{aligned}
\alpha(X \cdot Y) & =\alpha\{X, Y, E\}_{A}=g_{\imath}\{X, Y, E\}_{A}=\left\{g_{\tau}(X), g_{\tau}(Y), g_{\tau}(E)\right\}_{A^{\prime}} \\
& =\left\{g_{\tau}(X), g_{\tau}(Y), E^{\prime}\right\}_{A^{\prime}}=\alpha(X) \cdot \alpha(Y)
\end{aligned}
$$

for $X, Y \in A$. Hence $\alpha$ is an algebra isomorphism. This implies that $(A$, \langle\rangle$),\left(A^{\prime},\langle\rangle^{\prime}\right)$ are equivalent to each other.

q.e.d.

Remark 5.6. The proof for the effectivity of $\left(\mathfrak{g}_{A}, \rho_{A}\right)$ depends only on the fact that $L_{A} \ni \mathrm{id}_{A}$. Hence the OSGLA $\left(\mathfrak{g}=\sum \mathfrak{g}_{\mu}, \rho,\langle\rangle_{\mathfrak{p}}\right)$ associated with an $\operatorname{OJTS}(V,\{\},\langle\rangle)$ is effective if $L \ni \mathrm{id}_{V}$.

Let $\left(\mathfrak{g}=\sum \mathfrak{g}_{\mu}, \rho,\langle\rangle_{\mathrm{p}}\right)$ be the OSGLA associated with an OJTS $(V$, \{\}$,\langle\rangle)$. Assume that $L \ni \mathrm{id}_{V}$. Put $\nu=(0,-\mathrm{id}, 0) \in \mathfrak{p}$ and define an involutive automorphism $\theta$ of the complexification of $g$ by $\theta=\exp \operatorname{ad}(\pi \sqrt{-1} \nu)$. Then we have $\theta(X, F, Y)=(-X, F,-Y)$ for $(X, F, Y) \in \mathfrak{g}$. Hence $\theta$ leaves $\mathfrak{g}, \mathfrak{f}, \mathfrak{p}$ invariant. Set

$$
\begin{aligned}
\mathfrak{f}_{0} & =\mathfrak{H} \cap \mathfrak{g}_{0}=\left\{(0, F, 0) ; F \in L, F^{t}+F=0\right\} \\
\mathfrak{m} & =\mathfrak{H} \cap\left(\mathfrak{g}_{-1} \oplus \mathfrak{g}_{1}\right)=\{(X, 0, X) ; X \in V\} .
\end{aligned}
$$

Then $\mathfrak{f}_{0}, m$ are eigen spaces of eigen values $1,-1$ of the effective SLA $(\mathfrak{f}, \theta)$ respectively.

Let $K=\operatorname{Ad}_{\mathfrak{p}}(\mathfrak{f})=\left\{\left.\exp \operatorname{ad}(\mathfrak{f})\right|_{\mathfrak{p}}\right\}_{\text {gen }} \subset G L(\mathfrak{p})$ and set

$$
K_{0}=\{k \in K ; k(\nu)=\nu\} .
$$

Then $K_{0}$ is a closed subgroup of $K$. Since $(\mathfrak{g}, \rho)$ is effective by Remark 5.6 , the Lie algebras of $K, K_{0}$ are isomorphic to $\mathfrak{f}, \mathfrak{f}_{0}$ respectively. Note that $\theta K \theta^{-1}=K$ and that the Lie algebra of $K_{\theta}=\left\{k \in K ; \theta k \theta^{-1}=k\right\}$ is isomorphic to $\mathfrak{f}_{0}$ by the effectivity of $(\mathfrak{g}, \rho)$. Since 


$$
k \theta k^{-1}=\exp \operatorname{ad}(\pi \sqrt{-1} k(\nu))=\exp \operatorname{ad}(\pi \sqrt{-1} \nu)=\theta
$$

for $k \in K_{0}$, we have $K_{0} \subset K_{\theta}$. Hence the pair $\left(K, K_{0}\right)$ is a symmetric pair.

Let $K(\nu)$ be the $K$-orbit space of $\nu$. Then $K(\nu)$ is diffeomorphic to the homogeneous space $K / K_{0}$. The tangent space $T_{\nu}(K(\nu))$ is identified with the subspace $[m, \nu]=\{(X, 0,-X)\}: X \in V\}$. Note that the decomposition $\mathfrak{p}=\{(X, 0,-X) ; X \in V\} \oplus\left\{(0, F, 0) ; F \in L, F^{t}=F\right\}$ is orthogonal for \langle\rangle$_{\mathfrak{p}}$ by (5.9). Since $K$ acts isometrically for \langle\rangle$_{\mathrm{p}}$, the orbit space $K(\boldsymbol{\nu})$, with the metric induced from \langle\rangle$_{p}$, is a pseudo-riemannian symmetric space.

Theorem 5.7. (1) Let $\left(\mathfrak{g}=\sum \mathfrak{g}_{\mu}, \rho,\langle\rangle_{\mathfrak{p}}\right)$ be the OSGLA associated with an $\operatorname{OJTS}(V,\{\},\langle\rangle)$. Assume that $L \ni \mathrm{id}_{V}$. Then the orbit space $K(\nu)$ is a complete parallel submanifold of a pseudo-Euclidean space ( $\mathfrak{p}$, \langle\rangle$\left._{p}\right)$.

(2) Let $\left(\mathfrak{g}_{A}=\sum\left(\mathfrak{g}_{A}\right)_{\mu}, \rho_{A}, J_{p_{A}},\langle\rangle_{p_{A}}\right)$ be the HSGLA coming from an $\operatorname{OJA}(A,\langle\rangle)$. Then $L_{A} \ni \mathrm{id}_{A}$ and the orbit space $K(\nu)$ is a complete totally real parallel submanifold of a pseudo-Hermitian space $\left(\mathfrak{p}_{A}, J_{p_{A}},\langle\rangle_{p_{A}}\right)$. Moreover $K(\nu)$ is left invariant by the $S^{1}$-action: $\exp t J_{p_{A}}, t \in R$.

Proof. (1) We show that $K(\nu) \subset\left(\mathfrak{p},\langle\rangle_{\mathfrak{p}}\right)$ is parallel. Denote by $\sigma$ the second fundamental form of $K(\nu)$. Since the inclusion $K(\nu) \smile(\mathfrak{p}$, \langle\rangle$_{p}$ ) is equivariant, it is sufficient to see our claim at $\nu \in K(\nu)$. Identify the tangent space $T_{o}\left(K / K_{0}\right)$ at $o=K$ with the subspace $m$. Then the derivative $\nabla^{*} \sigma$ of $\sigma$ at $\nu$ is given by

$$
\left(\nabla^{*} \sigma\right)(A, B, C)=\{\operatorname{ad}(A) \operatorname{ad}(B) \operatorname{ad}(C) \nu\}_{\mathbb{8}_{0} \cap \mathfrak{p}}
$$

for $A, B, C \in \mathfrak{m}$, where $\{*\}_{\mathfrak{g}_{0} \cap \mathfrak{p}}$ denotes the $\mathfrak{g}_{0} \cap \mathfrak{p}$-component of $*$ for the decomposition $\mathfrak{p}=\mathfrak{g}_{0} \cap \mathfrak{p} \oplus\left(\mathfrak{g}_{-1} \oplus \mathfrak{g}_{1}\right) \cap \mathfrak{p}$ (See Ferus [5], Lemma 1 for the proof). Let $A=(X, 0, X), B=(Y, 0, Y), C=(Z, 0, Z)$ for $X, Y, Z \in V$. Then we have

$$
\begin{aligned}
\operatorname{ad}(\boldsymbol{A}) & \operatorname{ad}(\boldsymbol{B}) \operatorname{ad}(\boldsymbol{C}) \boldsymbol{\nu}=\operatorname{ad}(\boldsymbol{A}) \operatorname{ad}(\boldsymbol{B})(Z, 0,-Z) \\
& =\operatorname{ad}(\boldsymbol{A})(0,(1 / 2)\{L(Y, Z)+L(Z, Y)\}, 0) \\
& =(-(1 / 2)\{L(Y, Z)+L(Z, Y)\} X, 0,(1 / 2)\{L(Y, Z)+L(Z, Y)\} X)
\end{aligned}
$$

and thus $\left(\nabla^{*} \sigma\right)(A, B, C)=0$ for $A, B, C \in \mathfrak{m}$. This implies that $K(\nu)$ is a parallel submanifold.

(2) We show that $K(\nu) \subset\left(\mathfrak{p}_{A}, J_{p_{A}},\langle\rangle_{p_{A}}\right)$ is totally real. Since the inclusion is equivariant, it is sufficient to see our claim at $\nu \in K(\nu)$. Note 
that $T_{\nu}(K(\nu))=\{(X, 0,-X) ; X \in A\}, N_{\nu}(K(\nu))=\left\{\left(0, T_{X}, 0\right) ; X \in A\right\}$. Then we have $J_{p_{A}}(X, 0,-X)=[(E, 0, E),(X, 0,-X)]=\left(0, T_{X}, 0\right) \in N_{\nu}(K(\nu))$ for $X \in A$ $=V_{A}$. This implies that $K(\nu)$ is totally real in $\left(\mathfrak{p}_{A}, J_{p_{A}},\langle\rangle_{\mathfrak{p}_{A}}\right)$.

The other claims are obvious.

q.e.d.

Remark 5.8. Let $M^{r}$ be an $r$-dimensional complete totally real parallel submanifold of $\bar{M}^{r}(c), c \neq 0$, and $\hat{M}^{r+1} \subset N^{2 r+1}(c / 4) \subset E^{r+1}$ the complete inverse of $M^{r}$. Denote by $\tilde{\sigma}$ the second fundamental form of $\hat{M}^{r+1} \subset E^{r+1}$. Fix a point $p \in \hat{M}$ and put $A=T_{p}(\hat{M})$. Define a product - on $A$ by $X \cdot Y$ $=i \tilde{\sigma}(X, Y)$ for $X, Y \in A$. Let $\langle>$ be the restriction of the pseudo-riemannian metric \langle\rangle$_{E}$ into $A=T_{p}(\hat{M})$. Then $(A,\langle\rangle)$ is an OJA. The proof will be given in the second series of this paper.

\section{REFERENCES}

[1] B. Y. Chen-K. Ogiue, On totally real submanifolds, Trans. Amer. Math. Soc., 193 (1974), 257-266.

[2] P. Dombrowski, Differentiable maps into riemannian manifolds of constant stable osculating rank I, J. Reine Angew. Math., 274/275 (1975), 310-341.

[ 3 ] D. Ferus, Symmetric submanifolds of euclidean space, Math. Ann., 247 (1980), $81-93$.

[4. ] - Immersions with parallel second fundamental form, Math. Z., 140 (1974), 87-93.

[5] - Immersionen mit paralleler zweiter Fundamentalform: Beispiele und NichtBeispiele, Manuscripta Math., 12 (1974), 153-162.

[6] S. Helgason, Differential Geometry, Lie groups and Symmetric spaces, Academic Press, New York, 1978.

[ 7 ] S. Kobayashi and K. Nomizu, Foundations of Differential geometry I, II, Wiley (Interscience), 1963 and 1969.

[8] M. Koecher, "An elementary approach to bounded symmetric domains", Lect. Notes, Rice Univ., Houston, 1969.

[ 9 ] M. Kon, On some complex submanifolds in Kaehler manifolds, Canad. J. Math., 26 (1974), 1442-1449.

[10] H. Naitoh, Isotropic submanifolds with parallel second fundamental form in $P^{\mathrm{m}}(\mathrm{c})$, Osaka J. Math., 18 (1981), 427-464.

[11] —-, Totally real parallel submanifolds in $P^{\mathrm{n}}(\mathrm{c})$, Tokyo J. Math., 4 (1981) , 279-306.

[12] H. Naitoh and M. Takeuchi, Totally real submanifolds and symmetric bounded domains, Osaka J. Math., 19 (1982), 717-731.

[13] H. Nakagawa and Takagi, On locally symmetric Kaehler submanifolds in a complex projective space, J. Math. Soc. Japan, 28 (1976), 638-667.

[14] B. O'Neill, The fundamental equations of a submersion, Michigan Math. J., 131 (1966), 459-469.

[15] J. S. Pak, Planar geodesic submanifolds in complex space forms, Kodai Math. J., 1 (1978), 187-196.

[16] I. Satake, Algebraic structures of symmetric domains, Iwanami Shoten, Publishers and Princeton Univ. Press, 1981.

[17] W. Strübing, Symmetric Submanifolds of Riemannian Manifolds, Math. Ann., 245 (1979), 37-44. 
[18] M. Takeuchi, Polynomial representations associated with symmetric bounded domains, Osaka J. Math., 10 (1973), 441-475.

[19] —-, Parallel submanifolds of space forms, In: Manifolds and Lie groups (Papers in honor of Y. Matsushima), Birkhäuser, 1981.

Department of Mathematics

Yamaguchi University

Yamaguchi, 753 Japan 